\title{
The Higgs mass and the scale of new physics
}

\author{
Astrid Eichhorn, ${ }^{a, b}$ Holger Gies, ${ }^{c}$ Joerg Jaeckel, ${ }^{d}$ Tilman Plehn, ${ }^{d}$ Michael M. Scherer ${ }^{d}$ \\ and René Sondenheimer ${ }^{c}$ \\ ${ }^{a}$ Perimeter Institute for Theoretical Physics, \\ 31 Caroline St N, Waterloo, Ontario, N2L 2Y5, Canada \\ ${ }^{b}$ Blackett Laboratory, Imperial College London, \\ Prince Consort Rd, London, SW7 2AZ, United Kingdom \\ ${ }^{c}$ Theoretisch-Physikalisches Institut, Abbe Center of Photonics, \\ Friedrich-Schiller-Universität Jena, \\ Max-Wien-Platz 1, D-07743 Jena, Germany \\ ${ }^{d}$ Institut für Theoretische Physik, Universität Heidelberg, \\ Philosophenweg 16, D-69120 Heidelberg, Germany \\ E-mail: a.eichhorn@imperial.ac.uk, holger.gies@uni-jena.de, \\ jjaeckel@thphys.uni-heidelberg.de, plehn@uni-heidelberg.de, \\ scherer@thphys.uni-heidelberg.de, rene.sondenheimer@uni-jena.de
}

ABSTRACT: In view of the measured Higgs mass of $125 \mathrm{GeV}$, the perturbative renormalization group evolution of the Standard Model suggests that our Higgs vacuum might not be stable. We connect the usual perturbative approach and the functional renormalization group which allows for a straightforward inclusion of higher-dimensional operators in the presence of an ultraviolet cutoff. In the latter framework we study vacuum stability in the presence of higher-dimensional operators. We find that their presence can have a sizable influence on the maximum ultraviolet scale of the Standard Model and the existence of instabilities. Finally, we discuss how such operators can be generated in specific models and study the relation between the instability scale of the potential and the scale of new physics required to avoid instabilities.

Keywords: Renormalization Group, Effective field theories, Higgs Physics, Beyond Standard Model

ARXIV EPRINT: 1501.02812 


\section{Contents}

1 Introduction 1

2 Gauged Higgs-top model $\quad 3$

2.1 Standard Model running 3

2.2 Toy model 5

2.3 The issue of vacuum stability 8

2.4 Higgs mass bounds 12

$\begin{array}{lll}2.5 & \text { Fixed-point structure } & 17\end{array}$

3 Models for high-scale physics 18

$\begin{array}{lll}3.1 & \text { Heavy scalars } & 18\end{array}$

3.2 Heavy fermions 21

3.3 Non-perturbative new physics 22

4 Conclusions 23

$\begin{array}{ll}\text { A Light dark matter scalars } & 26\end{array}$

$\begin{array}{ll}\text { B Tunnelling } & 27\end{array}$

$\begin{array}{lr}\text { C Functional renormalization group } & 29\end{array}$

D Mapping between flow trajectories 33

E Modelling electroweak contributions to the full effective potential 35

\section{Introduction}

The recent discovery of a light, to current experimental precision elementary Higgs boson $[1-7]$ has a major effect on the entire field of particle physics. A narrow Higgs boson with a mass of $125 \mathrm{GeV}$ allows for an extrapolation of all fundamental interactions, except gravity, toward higher energy scales in terms of perturbative gauge theories. In particular, the renormalization group ( $R G)$ provides a link between the electroweak scale and more fundamental, higher energy scales. Renormalization group considerations for the Higgs sector typically provide two types of limits. An upper limit on the Higgs mass arises from the Higgs self-coupling becoming large (triviality bound) [8-15], while a lower limit arises from stability considerations of the Higgs potential [16-24]. The observed low Higgs mass suggests that the triviality bound will not be a problem for energy scales below the Planck scale. When it comes to the stability of the Higgs potential the situation is less clear [25-48]. 
As for any potential we refer to the Higgs potential as stable if it is bounded from below. We can attempt to link this condition to the sign of the quartic Higgs self-coupling evaluated at different energy scales [16-23], but the details of such a connection need to be tested carefully. Throughout this paper we will refer to this link as the perturbative or perturbatively renormalizable approach, because it typically relies on the assumption that all operators in the Higgs Lagrangian have at most mass dimension four. The great advantage of this approach is that the running couplings are known to high precision in the perturbative Standard Model, so we can compare its precise predictions to the measurements in the Higgs sector.

Alternatively, we can ask the question which additional operators, for example higher powers in the Higgs field, can render the Higgs vacuum stable and how such effective operators can be linked to particle physics models at the corresponding energy scales. Whereas the canonical irrelevance of higher-dimensional operators for low-energy observables is natural from a Wilsonian viewpoint, the presence of such operators is expected at high energies, once we approach the scales of an underlying theory. Perturbatively, such operators can for example appear as threshold effects from integrating out massive fields. Alternatively, they can arise from other non-trivial effects of a UV-completion. As such higher-dimensional operators do not appear in the usual, perturbatively renormalizable Higgs Lagrangian, we refer to this generalized approach as non-perturbative. Its main advantage is that it makes fewer assumptions about the Lagrangian at energies significantly above our experimental reach.

Based on the measured Higgs mass and top quark mass the perturbative analysis suggests that the electroweak Higgs vacuum might indeed not be stable. In the perturbative analysis the instability of the Higgs vacuum can be linked to the energy scale at which the Higgs quartic coupling turns negative. At the three-loop level this scale comes out around $10^{10} \mathrm{GeV}$, where the precise value depends sensitively on the Standard Model parameters. Several physical interpretations of this scale are possible: first, we can require that the Higgs potential be stable at all scales. New degrees of freedom would then have to appear below or at $10^{10} \mathrm{GeV}$, changing the renormalization group evolution, and rendering the potential stable [25-43]. We review one such approach based on a Higgs portal with a scalar dark matter candidate in the appendix $[49,50]$. Alternatively, we can require that the absolute value of the (negative) Higgs quartic coupling remains small. New physics only has to set in at the Planck scale, tunneling rates to the true vacuum have to be small, and our electroweak vacuum is meta-stable [45]. The third, less studied option [51-58] includes higher-dimensional operators which stabilize the Higgs vacuum beyond $10^{10} \mathrm{GeV}$. Such operators will appear in theories with physical cutoff or matching scales. We explore this third option.

The higher-dimensional operators can be linked to particle physics models, including heavy new particles [59]. Again, we choose additional scalars coupled through a Higgs portal. Unlike $\mathrm{TeV}$-scale new scalars, heavy particles do not rely on their renormalization group running over a wide range of scales to affect the vacuum structure. Instead, they change the full set of running Higgs self-couplings at high scales to modify physics at intermediate scales. 
Our starting point is a field theory with higher-dimensional operators but including only Standard Model fields. This theory is perturbatively non-renormalizable. Accordingly, there exists a finite UV-cutoff $\Lambda$ in the general spirit of the Standard Model as an effective theory. An appropriate tool for including all quantum fluctuations in the presence of higher-dimensional operators as well as a finite ultraviolet (UV)-cutoff is the functional renormalization group [60]. Our discussion of the stability of the Higgs potential proceeds in three steps:

1. To quantitatively study the stabilizing effects of higher-dimensional operators we need a model which reflects all essential features of the Standard Model. We construct and test such a model in sections 2.1 and 2.2 .

2. In this toy model we describe the stability conditions, compute the possible Higgs mass range, and analyze its fixed-point structure (sections 2.3-2.5).

3. Finally, in section 3 we study explicit models with additional heavy scalars and fermions and determine under which conditions they stabilize the Higgs vacuum, while yielding Higgs masses below the conventional stability bound.

In appendix A we discuss the effect of light states, in appendix B we summarize the computation of tunnelling rates, and in appendices $\mathrm{C}$ and $\mathrm{D}$ we review the impact of higher-dimensional operators and give a detailed link between the perturbative and nonperturbative approach to the Higgs-top renormalization group.

\section{Gauged Higgs-top model}

The aim of this paper is to investigate Higgs mass bounds and vacuum stability in the presence of higher-dimensional operators and a finite ultraviolet cutoff. In addition to the standard perturbative running, we use functional renormalization group (FRG) methods as a tool to compute the running of an extended set of operators. Let us first set up a toy model that allows us to study the essential features of the Standard Model in the context of vacuum stability.

As a starting point, we briefly recapitulate the main features of the Standard Model at one-loop level. In the introductory sections we use $H$ for the actual Higgs scalar, while $\varphi$ denotes a general real scalar field which can play the role of the Higgs field $H$ in our toy model. Once we arrive at our toy model which quantitatively reproduces the Standard Model, we will again use $H$ for the corresponding scalar Higgs field.

\subsection{Standard Model running}

The perturbative approach starts from the usual Higgs potential, generalized to an effective potential by allowing for a dependence on the momentum scale $k$ of all parameters. Including higher-dimensional operators it reads

$$
V_{\mathrm{eff}}(k)=\frac{\mu(k)^{2}}{2} H^{2}+\sum_{n=2} \frac{\lambda_{2 n}(k)}{k^{2 n-4}}\left(\frac{H^{2}}{2}\right)^{n}=\frac{\mu^{2}(k)}{2} H^{2}+\frac{\lambda_{4}(k)}{4} H^{4}+\frac{\lambda_{6}(k)}{8 k^{2}} H^{6}+\cdots,
$$


where we denote the scalar doublet as $(H / \sqrt{2}, 0)=((h+v) / \sqrt{2}, 0)$, with $h$ parametrizing the excitation above the expectation value $v$ and neglecting the Goldstone modes. In this form it is not at all clear whether a slightly negative $\lambda_{4}$ will lead to an unstable potential. This depends on the higher-dimensional couplings $\lambda_{6,8, \ldots}$ which, if sufficiently large, can obviously stabilize the Higgs potential for all $k<M_{\mathrm{Pl}}$.

Standard power counting shows that long-range observables are dominated by perturbatively renormalizable operators. Hence, one might think that higher-dimensional operators can generically be ignored from the outset. With the so-defined Standard Model the corresponding Lagrangian consists of all dimension- 4 operators. In that case the question of stability is usually linked to the sign of $\lambda_{4}$ defined in eq. (2.1). The beta function for any coupling $g$ is defined as $\beta_{g}=d g / d \log k$. In these conventions the one-loop renormalization group equations for the Higgs self-coupling $\lambda_{4}$, the top Yukawa $y$, and the strong coupling $g_{s}$ in the Standard Model read

$$
\begin{aligned}
\beta_{\lambda_{4}} & =\frac{d \lambda_{4}}{d \log k}=\frac{1}{8 \pi^{2}}\left[12 \lambda_{4}^{2}+6 \lambda_{4} y^{2}-3 y^{4}-\frac{3}{2} \lambda_{4}\left(3 g_{2}^{2}+g_{1}^{2}\right)+\frac{3}{16}\left(2 g_{2}^{4}+\left(g_{2}^{2}+g_{1}^{2}\right)^{2}\right)\right] \\
\beta_{y}=\frac{d y}{d \log k} & =\frac{y}{16 \pi^{2}}\left[\frac{9}{2} y^{2}-8 g_{s}^{2}-\frac{9}{4} g_{2}^{2}-\frac{17}{12} g_{1}^{2}\right] \\
\beta_{g_{s}}=\frac{d g_{s}}{d \log k} & =-\frac{g_{s}^{3}}{16 \pi^{2}}\left[11-\frac{2}{3} n_{f}\right] .
\end{aligned}
$$

The top Yukawa coupling is linked to the running top mass by $y=\sqrt{2} m_{t} / v$, while the Higgs mass is given by $m_{h}^{2}=2 \lambda_{4} v^{2}$ plus contributions from higher-dimensional operators. The couplings or the related running masses $m_{t}$ and $m_{h}$ can be translated into the corresponding pole or on-shell masses, for example to describe the kinematics of production and decay processes. The gauge couplings are $g_{s}$ for $\mathrm{SU}(3)_{c}, g_{2}$ for $\mathrm{SU}(2)_{L}$ and $g_{1}$ for $\mathrm{U}(1)_{Y}$. The number of fermions contributing to the running of the strong coupling is $n_{f}$. In the Standard Model setup of eq. (2.2) no explicit higher-dimensional operators occur. However, if they are generated by Standard Model particle loops, the effect of, e.g., an induced $\lambda_{6}$ coupling is included in the running of the renormalizable parameters at a higher loop order. We will return to a more precise discussion of this point in section 2.5.

To allow for an easy comparison of our toy model to the Standard Model we can translate the beta function for the Higgs self-coupling into the running Higgs mass, namely

$$
\beta_{m_{h}}=\frac{d m_{h}}{d \log k}=\frac{3}{8 \pi^{2} v^{2} m_{h}}\left[m_{h}^{4}+2 m_{h}^{2} m_{t}^{2}-4 m_{t}^{4}+\text { weak terms }\right] .
$$

Let us take a brief look at the essential features that arise from the renormalization group running given by eq. (2.2). Toward the UV, the negative contribution of the top Yukawa term drives the Higgs self-coupling $\lambda_{4}$ to small values and eventually through zero. Ignoring higher-dimensional operators, the Higgs potential seems to become unstable at large energy scales, before the stabilizing effect of the weak gauge coupling sets in at very high scales. This is the usual (meta-)stability issue in the perturbative setting. We will give a more detailed interpretation in section 2.3.

For the quantitative behavior of $\lambda_{4}$ in eq. (2.2) it is important that the top Yukawa coupling $y$ also decreases toward the ultraviolet under the influence of the strong coupling $\alpha_{s}$. 
Indeed, for the situation we want to investigate, $\lambda_{4}$ and $y$ both decrease toward the ultraviolet, and the loop contributions from those couplings to their running get smaller and smaller. At some point the contributions from the weak couplings and $y$ to the running of $\lambda_{4}$ become comparable in size. When their loop contributions almost cancel, the running of $\lambda_{4}$ becomes flat, $\beta_{\lambda_{4}} \approx 0$. Finally, in the deep ultraviolet the weak gauge couplings dominate and turn $\lambda_{4}$ back to positive values. This behavior is shown in the upper left panel of figure 1 [45].

Following this argument, the self-coupling $\lambda_{4}$ in the Standard Model appears to first turn negative and finally become positive again. From this behavior one could conclude that we live in a so-called meta-stable vacuum if the Higgs potential, including only operators to dimension- 4 is given by

$$
V(H) \approx \lambda_{4}(H) \frac{H^{4}}{4} \quad \text { with } \quad H \gg k_{\mathrm{EW}}
$$

at high field values. Here, it is assumed that the effective potential is well approximated by identifying the RG scale with the field amplitude, $\lambda_{4}(H) \equiv \lambda_{4}(k=H)$. This potential features two minima, our electroweak minimum and a global minimum at very large field values $H \gg M_{\mathrm{Pl}}$. The latter occurs far outside the region where the renormalization group equations of eq. (2.2) can be trusted. We therefore focus on stabilizing effects that set in below the Planck scale.

\section{$2.2 \quad$ Toy model}

In this section we set up a simple model that exhibits most of the essential features of the behavior of the running Standard Model couplings without inheriting the full gauge structure. First, we replace the Higgs field $H$ as part of an $\mathrm{SU}(2)$ doublet by a general real scalar field $\varphi$ featuring a discrete $\mathbb{Z}_{2}$ chiral symmetry. This ensures that no Goldstone modes alter the renormalization group flow in the symmetry-broken regime, just as in the Standard Model with the full gauge structure. In a simple Yukawa system without gauge degrees of freedom we already observe a similar perturbative flow toward negative $\lambda_{4}$ at high scales [54]. The running of the top Yukawa coupling to smaller values in the ultraviolet is included when we add an SU(3) gauge sector. Correspondingly, we investigate the Euclidean action defined at the UV-cutoff scale $\Lambda$

$$
S_{\Lambda}=\int d^{4} x\left[\frac{1}{4} F_{\mu \nu}^{a} F^{a \mu \nu}+\frac{1}{2}\left(\partial_{\mu} \varphi\right)^{2}+V_{\mathrm{eff}}(\Lambda)+i \sum_{j=1}^{n_{f}} \bar{\psi}_{j} \not D \psi_{j}+i \frac{y}{\sqrt{2}} \sum_{j=1}^{n_{y}} \varphi \bar{\psi}_{j} \psi_{j}\right],
$$

with an $\mathrm{SU}\left(N_{c}\right)$ gauge field $\left(N_{c}=3\right)$, a real scalar $\varphi$, Dirac fermions $\psi_{j}$ for $n_{f}$ flavors, the covariant derivative $D_{\mu}$ including the strong coupling $g_{s}$, and the effective scalar potential $V_{\text {eff. }}$ To avoid confusion, we emphasize that the Higgs potential $V_{\text {eff }}(\Lambda)$ is the bare potential from the Standard Model point of view. Embedding the Standard Model into a more fundamental theory, the Higgs potential becomes the effective potential of that theory, containing all effects of quantum fluctuations above $\Lambda$. We assume that $n_{y}<n_{f}$ of the fermion species are heavy and shall have a degenerate large Yukawa coupling $y$. The 

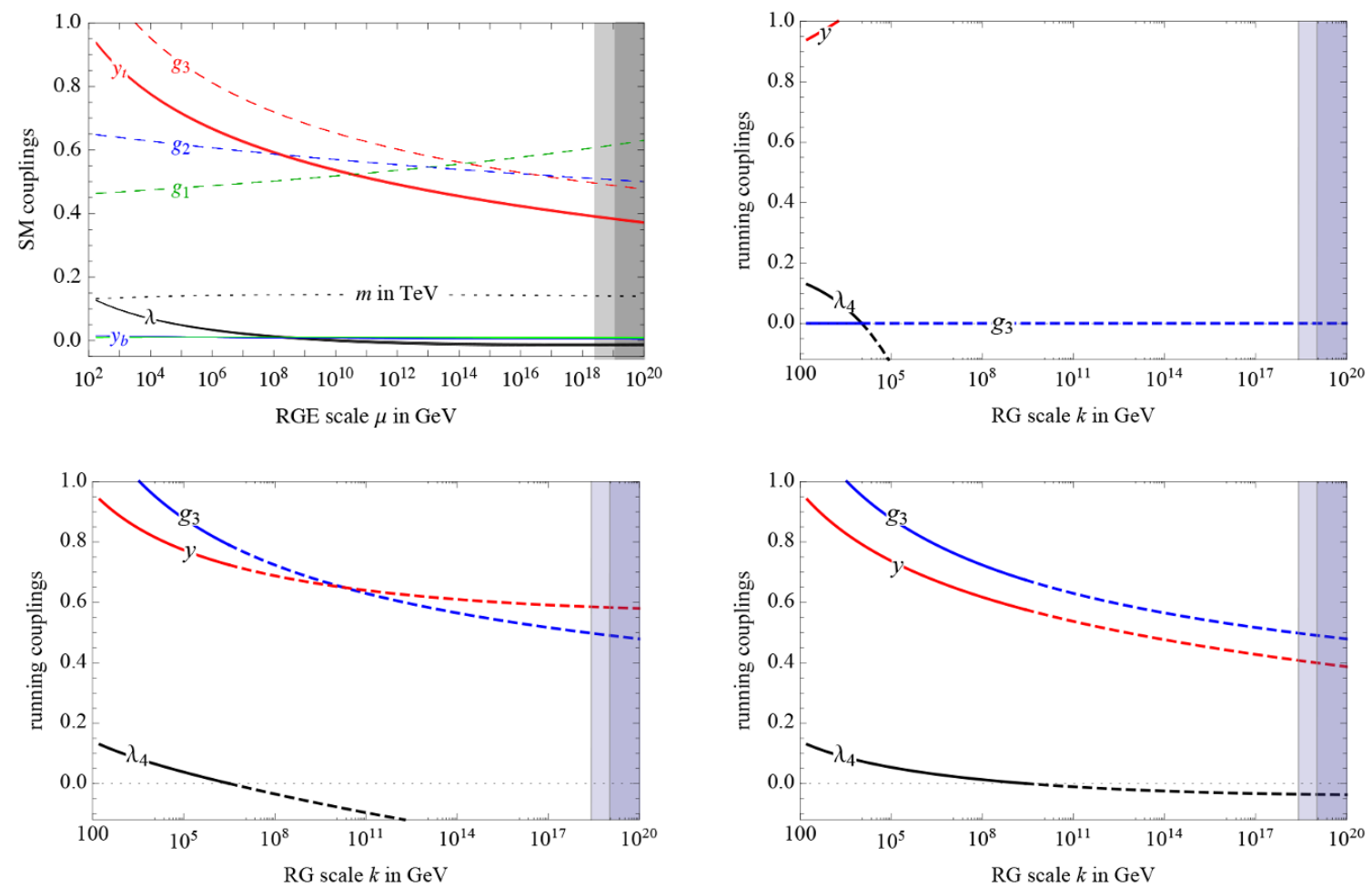

Figure 1. Upper left: running of SM couplings, figure taken from ref. [45]. Upper right to lower right: running of our toy model couplings after including the running strong coupling and electroweak coupling effects. Dashed lines indicate the regime where $\lambda_{4}(k)<0$, which in the perturbative approach defines the loss of vacuum stability.

remaining $n_{f}-n_{y}$ flavors have negligible Yukawa couplings. The effective scalar potential is now expanded in terms of dimensionless couplings $\lambda_{2 n}$ as

$$
V_{\mathrm{eff}}(k)=\frac{\mu(k)^{2}}{2} \varphi^{2}+\sum_{n=2} \frac{\lambda_{2 n}(k)}{k^{2 n-4}}\left(\frac{\varphi^{2}}{2}\right)^{n}
$$

This potential should be compared to the Standard Model potential shown in eq. (2.1). As part of the potential we study beta functions for the self-interaction $\lambda_{4}$ and the $\varphi^{6}$-coupling $\lambda_{6}$. Here we restrict ourselves to the first in an (infinite) series of possible higher-order couplings.

Because our toy model does not reflect the weak gauge structure of the Standard Model, the gauge couplings $g_{1}$ and $g_{2}$ do not appear. However, we know that in the Standard Model they have significant effects: first, the gauge couplings give a significant positive contribution to $\beta_{\lambda_{4}}$, balancing the negative top Yukawa terms for small values of $\lambda_{4}$; second, they decrease the Yukawa coupling in the UV, thereby also increasing $\lambda_{4}$ toward large scales. Since the variation of the weak coupling at large energy scales is modest, we account for its effects by including a finite contribution in the beta functions for $\lambda_{4}$ and $y$, 
parametrized by a fiducial coupling $g_{F}$ and numerical constants $c_{\lambda}, c_{y}$.

$$
\begin{aligned}
\beta_{\lambda_{4}} & =\frac{1}{8 \pi^{2}}\left[-n_{y} N_{c} y^{4}+2 n_{y} N_{c} y^{2} \lambda_{4}+9 \lambda_{4}^{2}-\frac{15}{4} \lambda_{6}+c_{\lambda} g_{F}^{4}\right] \\
\beta_{\lambda_{6}} & =2 \lambda_{6}+\frac{1}{16 \pi^{2}}\left[2 n_{y} N_{c} y^{6}+6 n_{y} N_{c} y^{2} \lambda_{6}-108 \lambda_{4}^{3}+90 \lambda_{4} \lambda_{6}\right] \\
\beta_{y} & =\frac{y}{16 \pi^{2}}\left[\frac{3+2 n_{y} N_{c}}{2} y^{2}-3 \frac{N_{c}^{2}-1}{N_{c}} g_{s}^{2}-c_{y} g_{F}^{2}\right] \\
\beta_{g_{s}} & =-\frac{g_{s}^{3}}{16 \pi^{2}}\left[\frac{11}{3} N_{c}-\frac{2}{3} n_{f}\right] .
\end{aligned}
$$

The scalar mass is given by $m_{\varphi}^{2}=2 \lambda_{4} v^{2}$, and the running top mass by $y=\sqrt{2} m_{t} / v$. To approximate the Standard Model we choose $n_{f}=6$ to account for the contribution of all flavors to the running of the strong gauge coupling. As long as we only keep the top quark contribution to the running of the Higgs quartic coupling, we set $n_{y}=1$. The expressions in eq. (2.7) reproduce the standard one-loop beta functions for our model if higher-dimensional operators are ignored [61].

We have verified that the simple modelling of the electroweak gauge boson effects in eq. (2.7) already provides a reasonable estimate. In a more self-consistent treatment, electroweak contributions to all running couplings $\mu^{2}, \lambda_{4}, \lambda_{6} \ldots$ of the Higgs potential appear. This is discussed in more detail in appendix E.

The running of the strong coupling and the top Yukawa coupling in our model agree exactly with that of the Standard Model given in eq. (2.2). Only the running of the scalar quartic vs Higgs quartic coupling is slightly different because of different numbers of degrees of freedom. To compare our toy model with the Standard Model we also write the beta function for the scalar quartic coupling in terms of the scalar mass as in eq. (2.3),

$$
\begin{aligned}
\beta_{m_{\varphi}} & =\frac{3}{8 \pi^{2} v^{2} m_{\varphi}}\left[\frac{3}{4} m_{\varphi}^{4}+2 m_{t}^{2} m_{\varphi}^{2}-4 m_{t}^{4}+\text { weak and } \lambda_{6} \text { terms }\right] \\
& =\frac{3}{8 \pi^{2} v^{2} m_{\varphi}}\left[\left(0.93 m_{\varphi}\right)^{4}+2 m_{t}^{2} m_{\varphi}^{2}-4 m_{t}^{4}+\text { weak and } \lambda_{6} \text { terms }\right] .
\end{aligned}
$$

The only difference is the self-interaction contribution to the running of the scalar or Higgs mass. This means that we can model the running of the top-Higgs sector if we slightly shift the scalar and top mass ratio at the per-cent level.

In figure 1 we illustrate our toy model with representative one-loop renormalization group flows in three steps:

1. in the upper left panel we show the full 3-loop Standard Model running from ref. [45] as reference;

2. in the upper right panel we include a scalar field and one heavy (top) quark, $n_{y}=1$, without any contributions from the gauge couplings (pure $\mathbb{Z}_{2}$ model);

3. in the lower left panel we add the effect of $\alpha_{s}$ on the running top Yukawa coupling; the strong coupling itself runs with $n_{f}=6$ active flavors;

4. finally, in the lower right panel we also include a constant fiducial coupling with $c_{\lambda}=9 / 16, c_{y}=97 / 30$ and $g_{F}=0.57$ to model the effect of the weak coupling. 
Since this choice of parameters allows us to quantitatively reproduce the behavior of the Higgs sector in the Standard Model, we refer to the corresponding scalar field given the appropriate model parameters as the 'Higgs scalar', i.e. $\varphi \rightarrow H$. For the flow trajectories we choose the running top mass such that $m_{t}\left(m_{t}\right)=164 \mathrm{GeV}$ [45], and correspondingly the Higgs mass at $m_{h}=2 \lambda_{4}\left(m_{t}\right) v^{2}=125 \mathrm{GeV}$. This means that we ignore the small difference between the running Higgs mass $m_{h}\left(m_{t}\right)$ and the measured Higgs pole mass. The running of the strong coupling satisfies $\alpha_{S}\left(m_{Z}\right)=0.1184$ at $m_{Z}=91 \mathrm{GeV}$ [45].

When we include the strong coupling in the bottom left panel of figure 1 the top Yukawa coupling decreases toward the ultraviolet. This allows for lower Higgs mass bounds from vacuum stability compared to the pure $\mathbb{Z}_{2}$ model [54]. With the additional approximate effects from the weak coupling we also reproduce the back-bending toward positive $\lambda_{4}$ in the deep ultraviolet, as it occurs in the Standard Model, shown in the bottom right panel of figure 1. The weak coupling term $c_{y}$ in the flow of the top Yukawa coupling is responsible for the faster decrease of the Yukawa coupling, such that it flows almost parallel to the strong gauge coupling. Thereby also the flow of $\lambda_{4}$ becomes flatter. The weak coupling contribution $c_{\lambda}$ to the running of the Higgs quartic coupling has a smaller impact at intermediate scales and induces the final back-bending of $\lambda_{4}$ close to the Planck scale.

\subsection{The issue of vacuum stability}

In this section we clarify some aspects of the relation between the perturbative and nonperturbative approaches. First we address a potential confusion whether the top loop stabilizes or de-stabilizes the potential. Second, in the perturbative approach the RG scale is replaced by the field value. In the non-perturbative approach both, the RG scale and the field value appear explicitly. Finally, in the perturbative approach one usually employs a massless regulator based on dimensional regularization. The question arises what happens in the presence of a finite, physical UV-cutoff.

Let us start by briefly recalling how the issue of vacuum stability arises and what role a finite cutoff plays. We refer to a potential as stable if it is bounded from below, otherwise it is unstable. A stable potential can exhibit several minima, where a local minimum might be meta-stable while the global minimum is stable. As argued above, the top loop is by far the dominant factor in the renormalization group evolution of the Higgs potential. Indeed, the whole issue of stability can already be understood qualitatively by considering the term $\beta_{\lambda_{4}}=-3 y^{4} /\left(8 \pi^{2}\right)+\cdots$ in eqs. (2.2) and (2.7). Integrating the $\beta$-function toward the ultraviolet, this term drives the quartic self-coupling to negative values at some high scale. This is the usual argument for the loss of stability in the Higgs potential if the Higgs potential is approximated by $V \approx \lambda_{4}(H) H^{4} / 4$.

This observation appears to be in conflict with non-perturbative lattice simulations [62, $63]$ and with arguments that the interacting part of the top loop is non-negative and cannot induce any instability in the presence of a finite UV-cutoff scale $\Lambda$ [55-57]. To understand this in more detail, let us consider a one-loop calculation of the effective potential at the electroweak scale $k \approx k_{\mathrm{EW}} \approx 0$, where we neglect $k_{\mathrm{EW}} \ll \Lambda$ when appropriate. As shown in ref. [55] the fermion determinant representing the top contribution to the effective potential 
for any finite cutoff $\Lambda$ can be written as

$$
\Delta V_{\text {top }}=-c_{2} \Lambda^{2} H^{2}+\text { positive terms, }
$$

where $c_{2}>0$. We start from a stable bare potential $V_{\mathrm{UV}}=V_{\mathrm{eff}}(\Lambda)$ of the kind shown in eq. (2.1) with quadratic and quartic Higgs terms. The top loop then gives us an approximate weak-scale potential

$$
V_{\mathrm{eff}}\left(k_{\mathrm{EW}}\right) \approx V_{\mathrm{UV}}+\Delta V_{\mathrm{top}}=\frac{\mu^{2}(\Lambda)-c_{2} \Lambda^{2}}{2} H^{2}+\frac{\lambda_{4}(\Lambda)}{4} H^{4}+\text { positive terms, }
$$

and will never lead to an unstable effective potential at the weak scale. A closer look at the contribution from the top loop below the cutoff scale $\Lambda$ reveals the formally sub-leading terms

$$
\begin{aligned}
\Delta V_{\mathrm{top}}= & -\frac{1}{4 \pi^{2}} \int_{0}^{\Lambda} d q q^{3} \log \left(1+\frac{y^{2} H^{2}}{2 q^{2}}\right) \\
= & -\frac{y^{2}}{16 \pi^{2}} \Lambda^{2} H^{2}+\frac{1}{64 \pi^{2}}\left[y^{4} H^{4} \log \left(1+\frac{2 \Lambda^{2}}{y^{2} H^{2}}\right)+2 y^{2} \Lambda^{2} H^{2}\right. \\
& \left.-4 \Lambda^{4} \log \left(1+\frac{y^{2} H^{2}}{2 \Lambda^{2}}\right)\right] \\
= & -c_{2} \Lambda^{2} H^{2}+c_{4} \frac{y^{4}}{4} H^{4} \log \frac{\Lambda}{k_{\mathrm{EW}}}+\ldots,
\end{aligned}
$$

where we normalize the contribution such that $\Delta V_{\text {top }}(H=0)=0$. In the last line we adopt the general notation of eq. (2.9). The additional logarithmic contribution to the quartic potential term contributes to the Higgs mass as [54, 64-66]

$$
\lambda_{4}\left(k_{\mathrm{EW}}\right)=\lambda_{4}(\Lambda)+c_{4} y^{4} \log \frac{\Lambda}{k_{\mathrm{EW}}} \approx \frac{1}{8} .
$$

This is nothing but the renormalization of the Higgs self-coupling. For a sufficiently large cutoff scale $\Lambda$ the above top contribution forces us to choose a negative high-scale value $\lambda_{4}(\Lambda)<0$ to obtain the measured Higgs mass.

While no instability in the effective potential is induced by the top loop, its contribution is such that we would have to choose an unstable potential at the ultraviolet scale $\Lambda$ to reproduce the measured Higgs mass in the infrared. The usual perturbative renormalization group evolution simply determines the value we would have to choose in the ultraviolet in the class of pure $H^{4}$-potentials and in that sense reflects the fact that we would have to choose an unstable UV-potential if we want to start at a very high scale $\Lambda$.

Stabilizing the UV-potential requires higher-dimensional operators. In this situation the stability of the UV-potential cannot be determined from the running of $\lambda_{4}$ alone, but requires the full UV-potential. As a simple example, we include a $\lambda_{6}$ term as in eq. (2.1),

$$
V_{\mathrm{UV}}=V_{\mathrm{eff}}(\Lambda)=\frac{\mu^{2}(\Lambda)}{2} H^{2}+\frac{\lambda_{4}(\Lambda)}{4} H^{4}+\frac{\lambda_{6}(\Lambda)}{8 \Lambda^{2}} H^{6} .
$$

As we have seen, $\lambda_{4}(\Lambda)$ is essentially fixed by the measured value of the Higgs mass in the infrared. For sufficiently large $\Lambda$ it is negative. Similarly $\mu^{2}(\Lambda)$ is fixed by requiring 

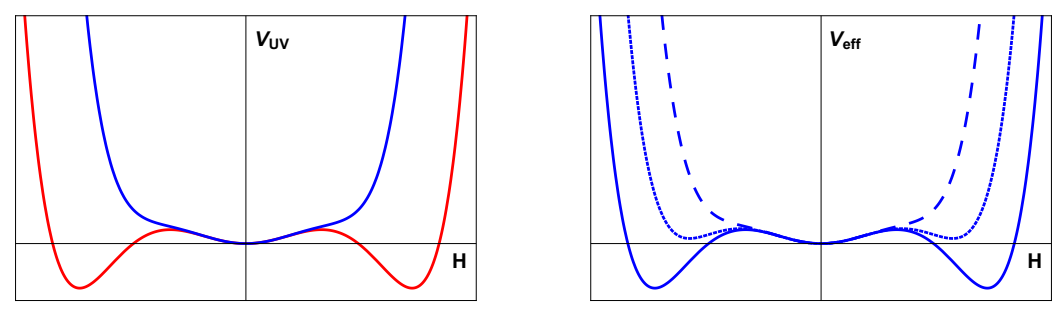

Figure 2. Left: sketch of the possible shape of $V_{\mathrm{UV}}$ with $\mu^{2}(\Lambda)>0$ but $\lambda_{4}(\Lambda)<0$. Potentials are stabilized by $\lambda_{6}(\Lambda)$ smaller (red) and larger (blue) than $\lambda_{4}^{2}(\Lambda) \Lambda^{2} /\left(3 \mu^{2}(\Lambda)\right.$ ), respectively. Right: sketch of different possible shapes of the effective potential $V_{\text {eff }}(k \approx 0)$ that may occur depending on the size of $\lambda_{6}(\Lambda)$, all corresponding to a stable UV-potential with a single minimum at $H \approx 0$ as the blue curve in the left panel. Note that the resolution of this sketch is not high enough to display the offset of the electroweak vacuum from $H=0$, the corresponding two minima appear as one at $H=0$.

$v=246 \mathrm{GeV}$ in the infrared. For the simple momentum-space cutoff regularization, it is typically large and positive, $\mu^{2}(\Lambda) \sim \mathcal{O}(0.01) \Lambda^{2}$. As expected, $\lambda_{6}(\Lambda)$ is the only new and free parameter. Its value is essentially undetermined by measurements in the infrared, because it is suppressed by the large scale $\Lambda$.

Choosing $\lambda_{6}(\Lambda)$ positive ensures that the UV-potential grows at large field values, making it a viable bare potential for a quantum field theory. In principle, this can be achieved with an arbitrarily small value of $\lambda_{6}(\Lambda)$. Since $\mu^{2}(\Lambda)>0$, the potential then has a qualitatively similar form to the red curve in the left panel of figure 2 with a local minimum at $H=0$ and a global minimum at a large field value $H \neq 0$. Choosing

$$
\lambda_{6}(\Lambda)>\lambda_{4}^{2}(\Lambda) \frac{\Lambda^{2}}{3 \mu^{2}(\Lambda)}
$$

removes the second minimum at $H \neq 0$ for negative $\lambda_{4}(\Lambda)$. The only minimum appears at $H=0$, as shown by the blue curve in figure 2 . In this paper we are mainly interested in this type of potentials.

Nevertheless, a stable UV potential with a single minimum at $H=0$ according to eq. (2.14) does not necessarily imply that the effective potential only features a single minimum. In the course of the RG flow, all types of behavior with a potential bounded from below shown in figure 3 do occur, depending on the choice of UV parameters.

Qualitatively, this behavior can be understood from the unique-vacuum condition in eq. (2.14), which can be applied to any scale $k$. While $\lambda_{6}(k)$ decreases rapidly for decreasing $k, \lambda_{4}$ grows at the same time and ultimately becomes positive. If $\lambda_{4}$ turns positive first, then the scale dependent potential is always stable during the RG evolution in our approximation, cf. left panel in figure 3. On the other hand, if eq. (2.14) is violated first, a second minimum appears and the minimum at $H=0$ can become meta-stable for some range of RG scales, cf. right panel in figure 3. However, a reliable (meta-)stability analysis of the full potential will require to go beyond the simple polynomial expansion of the effective potential around one minimum.

For completeness let us note what happens when we continue to run to lower energy scales. Independently of the high-scale behavior discussed above, $\mu^{2}(k)$ drops below zero 

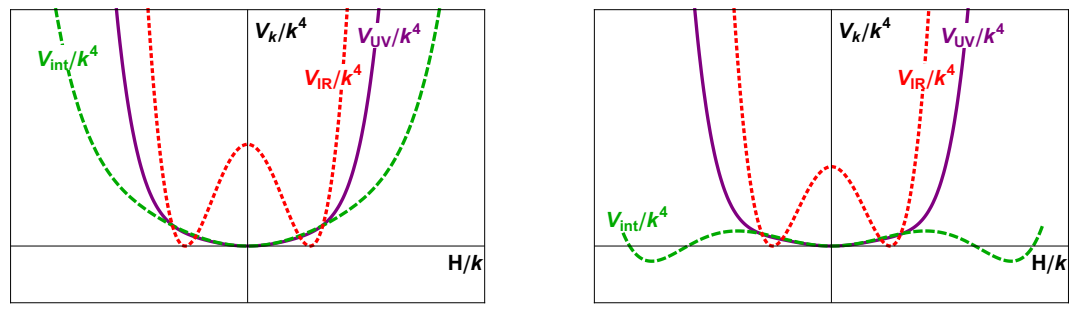

Figure 3. Starting from a stable potential in the UV different flows to the IR potential with the electroweak minimum are possible. In the left panel we show a situation where the potential remains stable during the whole flow from $k=\Lambda$ to the point where the electroweak minimum forms. This corresponds to region I in figure 6 . For smaller initial values of $\lambda_{6}$ corresponding to region II in figure 6, we observe a meta-stable behavior of the scale dependent potential already at intermediate values of $k$, which then disappears. The electroweak symmetry breaking minima are not formed until later. We call this behavior pseudo-stable.

near the electroweak scale. For these low scales $\lambda_{4}$ is always positive and the interplay with the negative $\mu^{2}(k)$ results in a finite vacuum expectation value $v$. In the presence of higher-dimensional operators, we rather generically find RG flows that interconnect a stable UV-potential with a minimum at $H=0$ with stable effective potentials in the infrared and a global electroweak minimum at $H=v$. If the flow passes through a finite regime where our polynomial approximation looks meta-stable, we refer to the scenario as pseudo-stable. In that case a full stability analysis requires a detailed non-perturbative analysis of the effective potential.

Our discussion shows that we have to distinguish between the stability of the UVpotential where no quantum fluctuations below $\Lambda$ are taken into account and that of the effective potential with all fluctuations included. In principle the UV potential and the effective potential can have quite different shapes as sketched in the left and right panels of figure 2. In the approximation $V \sim \lambda_{4}(H) H^{4} / 4$, this difference cannot be accounted for properly: on the one hand the running quartic coupling defines the UV-potential as $V(\Lambda)=\lambda_{4}(\Lambda) H^{4} / 4$. On the other hand, the identification $k \rightarrow H$ is assumed to be a good approximation for the effective potential. This means that one and the same function $\lambda_{4}(k)$ determines the stability of the UV-potential and the stability of the effective potential. The presence of higher-dimensional operators influences both aspects: at the UV-scale, they can modify the UV-potential in a rather general way. Successively, they can contribute to the RG flow of the renormalizable operators for some range of scales. The functional renormalization group takes these aspects into account by describing a scale-dependent effective potential $V_{\text {eff }}(k ; H)$, depending on both the scale $k$ as well as the field value, such that

$$
V(k=\Lambda ; H)=V_{\mathrm{UV}} \quad \text { and } \quad V(k=0 ; H)=V_{\mathrm{eff}} .
$$

In this manner, the (meta-)stability properties of the effective potential can be followed in a scale-dependent manner.

Finally, let us note another subtlety in the comparison between the perturbative and non-perturbative approaches. The perturbative approach usually relies on a massindependent regularization scheme. To avoid large threshold effects one should therefore 
stop the running of the couplings at the appropriate mass scales. Most of the relevant masses are proportional to $H$. This suggests to approximate

$$
V_{\text {eff }}=V(k=H, H) \approx \frac{1}{4} \lambda_{4}(H) H^{4},
$$

as is usually done in the perturbative approach. In eq. (2.12) we have already identified the running Higgs self-coupling at the scale $k_{\mathrm{EW}}$. Using the identification $k=H$ we essentially reproduce the first term in the square brackets of the second line of eq. (2.11). Let us check this explicitly,

$$
\begin{aligned}
V_{\mathrm{eff}}\left(k_{\mathrm{EW}}\right) \approx & V_{\mathrm{UV}}+\Delta V_{\mathrm{top}} \\
= & \frac{\mu^{2}(\Lambda)-c_{2} \Lambda^{2}}{2} H^{2}+\frac{1}{64 \pi^{2}}\left[y^{4} H^{4} \log \left(1+\frac{2 \Lambda^{2}}{y^{2} H^{2}}\right)+2 y^{2} \Lambda^{2} H^{2}\right. \\
& \left.-4 \Lambda^{4} \log \left(1+\frac{y^{2} H^{2}}{2 \Lambda^{2}}\right)\right]+\frac{\lambda_{4}(\Lambda)}{4} H^{4} \\
= & \frac{\mu^{2}\left(k_{\mathrm{EW}}\right)}{2} H^{2}+\frac{1}{64 \pi^{2}}\left[y^{4} H^{4} \log \left(1+\frac{2 \Lambda^{2}}{y^{2} H^{2}}\right)+2 y^{2} \Lambda^{2} H^{2}\right. \\
& \left.-4 \Lambda^{4} \log \left(1+\frac{y^{2} H^{2}}{2 \Lambda^{2}}\right)\right]+\frac{\lambda_{4}(\Lambda)}{4} H^{4} \\
= & \frac{\mu^{2}\left(k_{\mathrm{EW}}\right)}{2} H^{2}+\frac{1}{4}\left[\lambda_{4}(\Lambda)+\frac{y^{4}}{8 \pi^{2}} \log \left(\frac{\Lambda}{H}\right)+\frac{y^{4}}{32 \pi^{2}}-\frac{y^{4}}{16 \pi^{2}} \log \left(\frac{y^{2}}{2}\right)\right] H^{4} \\
& +\mathcal{O}\left(H^{4} \frac{H^{2}}{\Lambda^{2}}\right) \\
= & \frac{1}{4} \lambda_{4}(k=H) H^{4}+\frac{1}{8 \pi^{2}}(\text { finite in } \Lambda) H^{4}+\mathcal{O}\left(H^{4} \frac{H^{2}}{\Lambda^{2}}, H^{4} \frac{\mu^{2}\left(k_{\mathrm{EW}}\right)}{H^{2}}\right) .
\end{aligned}
$$

However, $V_{\text {eff }}=\lambda_{4}(H) H^{4} / 4$ only holds for $H \gg v \sim\left|\mu\left(k_{\mathrm{EW}}\right)\right|$ and $\Lambda \gg H$. This means that in the presence of a finite, physical UV-cutoff $\Lambda$ the perturbative running of $\lambda_{4}(H)$ provides a good approximation of the effective potential only for field values $H \ll \Lambda$. Indeed, using the perturbative running for a cutoff-free mass-independent regularization scheme can make the effective potential appear to be unstable beyond $H \sim \Lambda$ even when it is not $[55,62,63]$. Essentially, this results from a breakdown of the expansion in powers of $H / \Lambda$ when going from the second to the third line in eq. (2.17). For the use of the perturbative approach we therefore always have the condition that the field values - more generally any scale under consideration - are smaller than any physical cutoff.

\subsection{Higgs mass bounds}

When it comes to stability considerations, the main novel feature of our renormalization group flows is the presence of a second model parameter, $\lambda_{6}$, as a representative of general higher-dimensional operators. In the absence of $\lambda_{6}$ the stability condition for the bare potential, $\lambda_{4}(\Lambda)=0$, at the so-defined ultraviolet cutoff scale $\Lambda$ constitutes the lowest viable choice for the quartic coupling. Positive higher-dimensional couplings in the ultraviolet can allow for negative values of the Higgs quartic coupling and yet a stable potential. A negative quartic coupling in the ultraviolet also lowers the infrared quartic coupling value, leading to a stable potential with a Higgs mass below the conventional lower bound obtained when neglecting $\lambda_{6}$. 

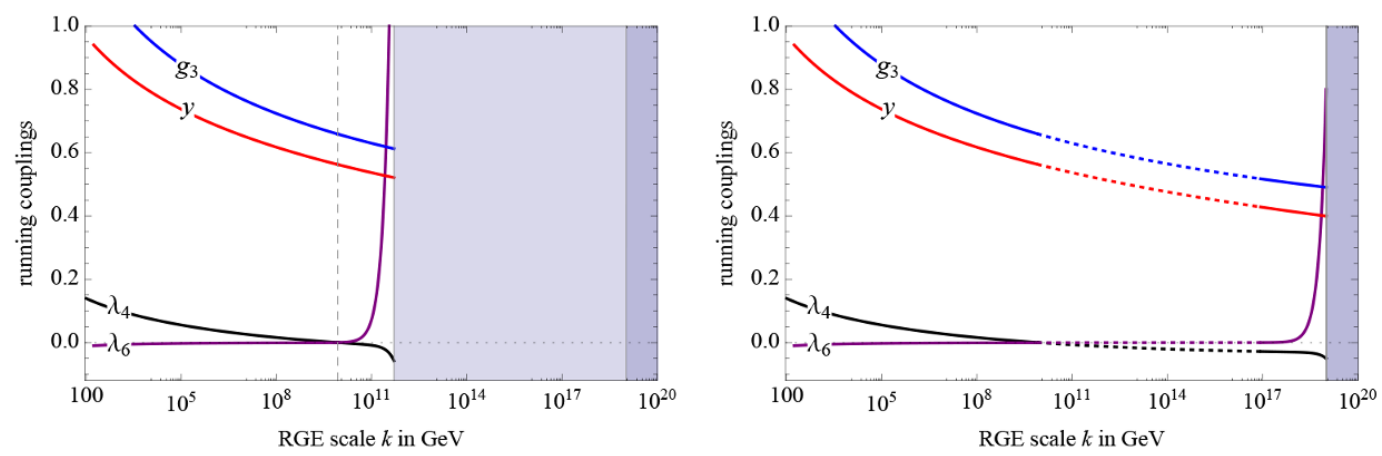

Figure 4. Running couplings including $\lambda_{4}$ and $\lambda_{6}$, corresponding to eq. (2.7) with $m_{h}=125 \mathrm{GeV}$. The bare potentials satisfy the stability condition of eq. (2.14) and thus remain bound from below at all lower scales. Left: $\lambda_{4}(\Lambda)=-0.058$ and $\lambda_{6}(\Lambda)=2.0$ and $\Lambda=5 \cdot 10^{11} \mathrm{GeV}$. Right: $\lambda_{4}(\Lambda)=-0.050$ and $\lambda_{6}(\Lambda)=0.8$ and $\Lambda=10^{19} \mathrm{GeV}$.

Let us now consider two examples for the influence of $\lambda_{6}>0$ as shown in figure 4 . In the first case, corresponding to the left panel of the figure we assume a relatively large UV-value of $\lambda_{6}$. At first, the effect of this term is sizable and therefore the RG flow deviates significantly from the usual perturbative scenario. However the canonical dimension of $\lambda_{6}$ implies that it quickly decreases toward the infrared, while $\lambda_{4}$ increases. At some scale the combined renormalization group flow reaches the point $\lambda_{6} \approx 0$ and $\lambda_{4} \approx 0$. This setup coincides with the ultraviolet starting point of the perturbative approach neglecting $\lambda_{6}$ and marks the boundary of stability. The infrared regime to the left of the dashed line in the left panel, where $\lambda_{4}$ again reaches positive values, is basically identical to the usual perturbative scenario. This means that due to higher-dimensional couplings our scenario features a significantly increased cutoff scale, while reaching the same values for the Higgs mass, quartic self-coupling, and top mass in the infrared. The naive upper limit on the validity of the model near $10^{10} \mathrm{GeV}$ (dashed line) is replaced by a higher scale $\sim 10^{12} \mathrm{GeV}$, at the expense of a nontrivial running of higher-dimensional operators. In between these two scales an effective field theory description within the model is still possible, while true new physics would presumably have to set in beyond $10^{12} \mathrm{GeV}$, in order to control the strong running of the scalar couplings.

In the second scenario, shown in the right panel of figure 4 , we choose a smaller value of $\lambda_{6}>0$ and a much larger cutoff scale $\Lambda$. In spite of this, the UV-potential at $\Lambda$ is still stable with a global minimum at $H=0$. As we have chosen a significantly larger cutoff scale $\Lambda, \lambda_{6}$ becomes smaller than required by the unique-vacuum condition eq. (2.14) before $\lambda_{4}$ reaches positive values. This implies an intermediate pseudo-stable region. The existence of this intermediate region indicated by dashed lines is a characteristic of the pseudo-stable scenario mentioned above. In our simple polynomial expansion, the scaledependent effective potential develops a second minimum and $H=0$ seems to be only a meta-stable minimum for intermediate RG scales. Our analysis based on the functional $\mathrm{RG}$ as outlined in appendix $\mathrm{C}$ going beyond the simple perturbative arguments indicates that this behavior should be assessed critically. We tentatively consider it an artifact of the polynomial expansion of the effective potential around one minimum. The functional 

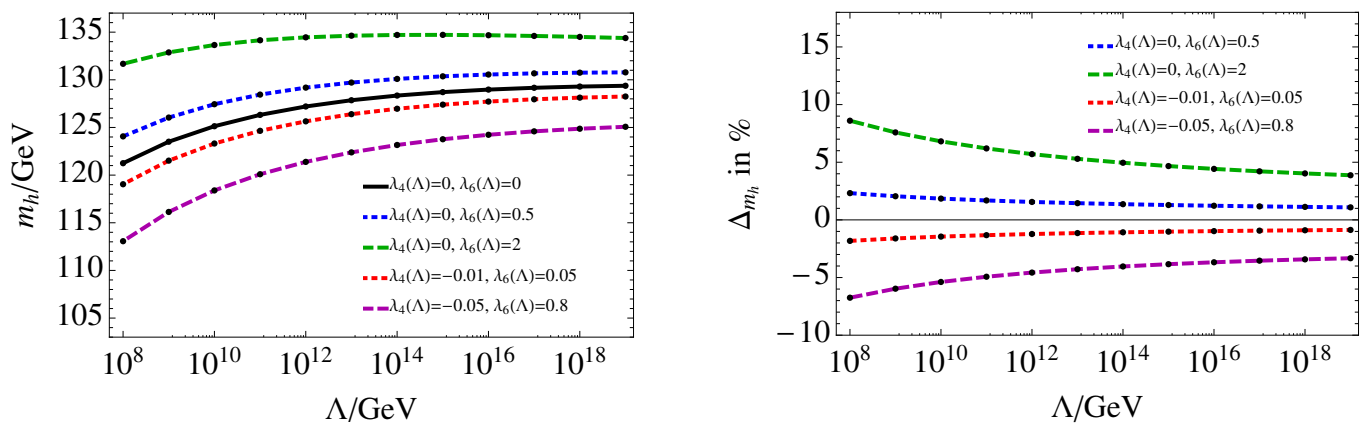

Figure 5. Infrared value of the Higgs mass (left) and the relative shift $\Delta_{m_{h}}$ measuring the departure from the conventional lower bound (right), as a function of the UV-cutoff $\Lambda$. We show different UV boundary conditions as indicated in the plot.

RG provides us with a $\beta$-function for the whole potential, eq. (C.3). We evaluate it in a polynomial expansion about an expansion point $H=0$ at high scales and $H=v$ near the weak scale. After integrating the flow we check whether the solution for the scale-dependent effective potential satisfies this $\beta$-function. Whereas the accuracy of the solution is indeed at the level of machine precision near the expansion point, the accuracy depletes away from this point. The convergence radius of this expansion is typically of the order of $m_{h}$ [54]. In particular, near the apparent new minima the polynomial expansion should not be trusted. Reliable statements about the stability of the effective potential in pseudo-stable scenarios require a dedicated analysis of the $\mathrm{RG}$ flow of the effective potential.

An improved functional RG analysis according to appendix $\mathrm{C}$ also clarifies the following apparent problem: in the infrared, the $\lambda_{6}$ coupling runs negative seemingly indicating a global instability. This is prevented by even higher-operators $\sim H^{8}$, which are taken into account in our improved functional RG analysis that we employ to determine the stability regions shown in figure 6 discussed below. All our explicit solutions for the scale-dependent effective potential are bounded from below.

Let us now examine the effects of $\lambda_{6}$ on the stability bounds for the Higgs mass. To derive Higgs mass bounds we first focus on a stable UV-potential with a global minimum at vanishing field values. In the absence of higher-dimensional couplings, the lower Higgs mass bound can be determined by requiring the quartic coupling to vanish at the cutoff scale $\Lambda$. Higher-dimensional couplings at the scale $\Lambda$ can take values of $\mathcal{O}(1)$ in units of the cutoff scale. Indeed, this is the generic situation that one would expect when examining an effective field theory such as the Standard Model close to its cutoff scale. In the left panel of figure 5 we first confirm that the observed Higgs mass around $125 \mathrm{GeV}$ corresponds to the ultraviolet boundary condition $\lambda_{4}=0$ at $\Lambda \approx 10^{10} \mathrm{GeV}$. Shifting this boundary condition closer to the Planck scale and hence allowing for a fully stable potential would require a Higgs mass close to $130 \mathrm{GeV}$ with a fixed top mass [44]. We also show several choices of ultraviolet boundary conditions. Those which predict smaller physical Higgs masses than the choice $\lambda_{i}(\Lambda)=0$ allow us to increase the ultraviolet cutoff. According to eq. (2.14) a viable choice for a UV-stable potential including higher-dimensional operators is for example $\lambda_{4}=-0.05$ and $\lambda_{6}=0.8$. The corresponding Higgs mass stays below the 
conventional lower bound for all values of $\Lambda$ and indeed gives a stable potential at the Planck scale, with a dip into a pseudo-stable regime at intermediate scales.

As described in detail in section 2.2 our calculation of the Higgs mass relies on a numerically convincing, but nevertheless a toy model. Special care is required when translating the computed shift of the Higgs mass at fixed cutoff to the Standard Model. We nevertheless conclude that shifts at the level of $1-5 \%$ seem viable. To see this we study the relative shift in the Higgs mass between the perturbative Standard Model and including the dimension- 6 self-coupling in figure 5 . It is given by

$$
\Delta_{m_{h}}(\Lambda)=\frac{m_{h}^{\left(\lambda_{i}(\Lambda) \neq 0\right)}-m_{h}^{\left(\lambda_{i}(\Lambda)=0\right)}}{m_{h}^{\left(\lambda_{i}(\Lambda)=0\right)}} .
$$

The dependence on $\Lambda$ is induced by the choice of ultraviolet cutoff scale at which we define the boundary conditions for $\lambda_{4}$ or $\lambda_{6}$. Negative values imply that the Higgs mass resulting from finite ultraviolet values of $\lambda_{4}$ and $\lambda_{6}$ lies below the conventional lower bound without exhibiting instabilities in the potential in the UV or at the weak scale. We emphasize, however, that large negative $\Delta_{m_{h}}$ go along with pseudo-stable scenarios that deserve more detailed investigations.

For all choices of UV-couplings we observe that $\left|\Delta_{m_{h}}\right|$ decreases with increasing $\Lambda$. This can be understood from the following argument [68-70]: including higher-dimensional couplings can allow us to extend the renormalization group flow toward the ultraviolet by several orders of magnitude, i.e. the cutoff scale is increased beyond the naive estimate determined by $\lambda_{4}(\Lambda)=0$. This corresponds to shifting the curve $m_{h}(\Lambda)$ in the left panel of figure 5 to the left by some amount $\Delta \Lambda$. The cutoff dependence of $m_{h}(\Lambda)$ flattens toward higher cutoff scales, thus such a shift is less effective for large cutoffs, and correspondingly $\Delta_{m_{h}}$ decreases in this regime.

While the achieved shifts in the allowed Higgs masses seem rather small, we emphasize that with present uncertainties in the experimental input parameters absolute stability of the Higgs potential without $\lambda_{6}$ terms is disfavored at $\sim 3 \sigma$ [45]. Our shift of the Higgs mass limit should be viewed as a shift in the central value which significantly reduces the tension between the measured data and the possibility that the Higgs potential is stable up to the Planck scale.

So far, we have restricted ourselves to absolutely stable bare potentials with a minimum at vanishing field values. Next, we turn to bare potentials bounded from below, but showing two minima. Presumably, this property also holds for the effective potential. However, since we employ a polynomial expansion of the potential we can only study the renormalization group flow around the electroweak minimum reliably. Future studies based on a different expansion will have to shed further light on the global renormalization group flow of the effective potential.

As already discussed in section 2.3, even a very small positive value of $\lambda_{6}$ is sufficient to ensure stability. However, the point $H=0$ is typically only meta-stable in this case. The phenomenological viability of this scenario requires that tunneling between the minima is sufficiently slow. To ensure this we enforce the conservative choice (cf. appendix B),

$$
\lambda_{4}(k)>-0.052, \quad \forall k .
$$




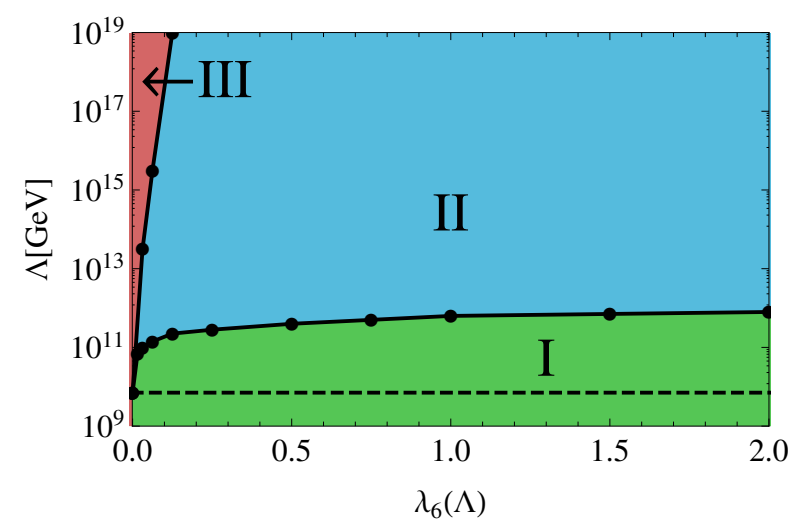

Figure 6. Different stability regions as a function of $\Lambda$ and $\lambda_{6}$. In region I (green) the potential is stable everywhere; in region II (blue) the UV-potential is stable, while the potential is only pseudo-stable for intermediate scales $v<k<\Lambda$; in region III (red) the UV-potential violates the unique-minimum condition. Our simple model includes the effects of the electroweak gauge bosons only in the running of $\lambda_{4}$. In appendix E, we model the electroweak contributions to the full Higgs potential. The corresponding region III is then somewhat larger, cf. figure 12 .

With this choice, the limit therefore essentially reduces to the usual longevity limit for the meta-stable region as obtained in the absence of any $\lambda_{6}$.

Our numerical study based on a more advanced approximation as described in appendix $\mathrm{C}$ shows that already with moderately small values $\lambda_{6} \approx 0.25$ the cutoff scale can be increased by approximately two orders of magnitude while retaining the full stability of the electroweak vacuum. This can be seen from the green region I in figure 6 . Increasing the possible cutoff scales by further orders of magnitude is difficult since there is a strongly infrared-attractive pseudo-fixed point at $\left|\lambda_{6}\right| \approx 0$ for those scales, see section 2.5 below. Next, there is a large pseudo-stable region (blue) where the UV-potential as well as the low-energy effective potential are stable, but our polynomially expanded potentials exhibit further minima at intermediate scales. As already explained, this is beyond the strict validity of our approximation and further studies are needed. Already relatively small values of $\lambda_{6}$ are sufficient to stabilize the UV-potential - although not the minimum at $H=0$ - up to the Planck scale, since $\lambda_{4}$ is negative, but its absolute value remains quite small. Finally, in the red region III the UV-potential is already meta-stable, as might be the effective potential. In this region the cutoff can easily take values beyond the Planck scale even for tiny values of $\lambda_{6}$, because the tunneling rates are always small enough to guarantee the longevity of the electroweak vacuum.

If we are well into region I, our RG flow indicates that the effective potential at $k \approx 0$ is stable. Our approximation should be reliable in this region. The fate of the pseudo-stable region II is not quite as clear. The appearance of the second minimum at intermediate values of $k$ hints at the possibility that the effective potential at $k \approx 0$ might be metastable. However, to fully establish this feature requires an approximation that goes beyond the local polynomial expansion we have used. Finally, in region III it is likely that the effective $k \approx 0$ potential will be meta-stable. 

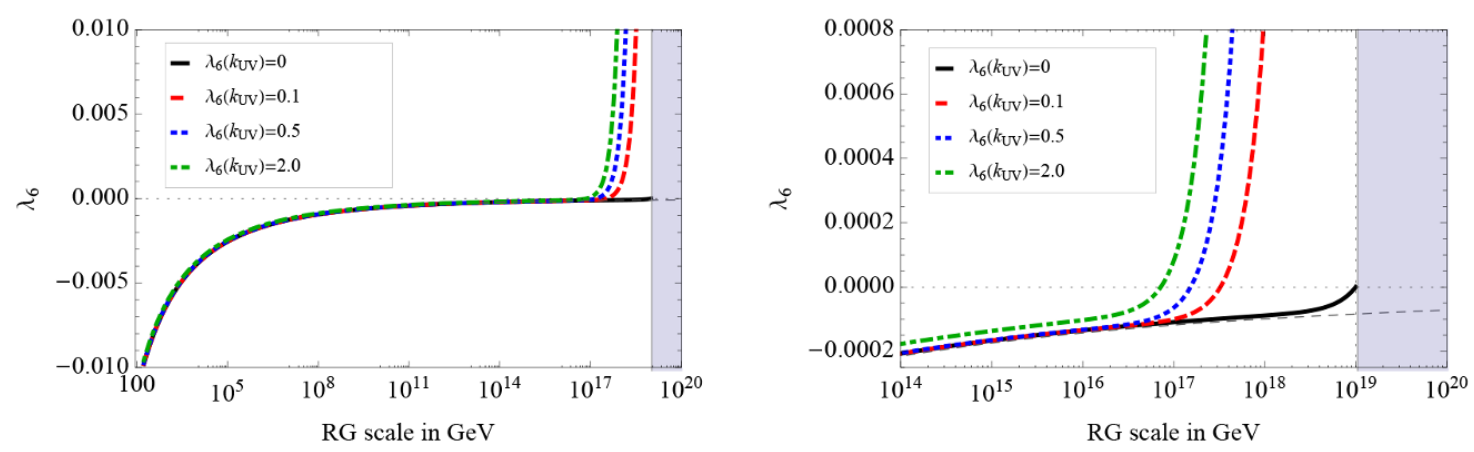

Figure 7. Running $\lambda_{6}(k)$ along RG trajectories with $\lambda_{6}\left(k_{\mathrm{UV}}\right)=0$ (black solid), $\lambda_{6}\left(k_{\mathrm{UV}}\right)=0.1$ (red dashed), $\lambda_{6}\left(k_{\mathrm{UV}}\right)=0.5$ (blue dotted), $\lambda_{6}\left(k_{\mathrm{UV}}\right)=2$ (green dot-dashed) and the pseudo-fixed point (thin grey dashed).

\subsection{Fixed-point structure}

To understand the behavior of higher-order couplings below the cutoff scale $\Lambda$, it is useful to analyze the beta functions in a little more detail. For couplings $\lambda_{6}$ and higher, they have the form

$$
\beta_{\lambda_{n}}=(n-4) \lambda_{n}+\eta \lambda_{n}+c .
$$

The first term reflects the canonical dimension of the operator. The second term is a generalized anomalous dimension term, i.e., $\eta$ is independent of $\lambda_{n}$. We also include contributions which are strictly speaking not an anomalous dimension, but are nevertheless linear in the coupling. For example, $\beta_{\lambda_{6}}$ contains such a term scaling like $\lambda_{4} \lambda_{6}$. Finally, $c$ is a contribution from other couplings and hence independent of $\lambda_{n}$. For instance, top quark fluctuations contribute proportional to $y_{t}^{n}$. The beta function for $\lambda_{6}$ thus features terms at most linear in $\lambda_{6}$. It is straightforward to find a pseudo-fixed point

$$
\begin{aligned}
\lambda_{6 *}=-\frac{c}{2+\eta}, \quad \text { with } \quad c & =\frac{2 n_{y} N_{c} y^{6}-108 \lambda_{4}^{3}}{16 \pi^{2}}, \\
\eta & =\frac{6 n_{y} N_{c} y^{2}+90 \lambda_{4}}{16 \pi^{2}},
\end{aligned}
$$

where the values for $c$ and $\eta$ can be read off from eq. (2.7). The pseudo-fixed point shows a remarkably small scale dependence, since the running of $\lambda_{4}$ and $y$ is also comparatively small. In fact, the ratio $\lambda_{4} / y^{2}$ also approaches a fixed point [68-70]. We neglect a possible contribution from $\lambda_{8}$. Because the anomalous dimension $\eta$ is small, the canonical dimension renders the fixed point strongly infrared attractive. In figure 7 we show that arbitrary starting values for $\lambda_{6}$ quickly converge toward the pseudo-fixed point. At low scales, $\lambda_{6}$ follows the rapidly growing top Yukawa coupling. As expected from the sign of the fermionic contribution in eq. (2.21), $\lambda_{6}$ becomes negative. This does not signal an instability, as higher-dimensional couplings also follow the value dictated by the Yukawa coupling and the fermion determinant contributes positively to the effective potential. The stability of the effective potential including top quark effects is thus guaranteed by our finite ultraviolet cutoff. 
In the perturbative approach, the explicit $\lambda_{6}$ dependence is not taken into account. However, some of the corresponding effects are encoded in higher loop diagrams [71]. This can be accounted for by setting $\lambda_{6}$ to its running pseudo-fixed point value [68-70]. In figure 7 we also show the running fixed-point value from eq. (2.7), i.e. the effective value of $\lambda_{6}$ included in loop effects in our definition of $\beta_{\lambda_{4}}$. To understand why the $\lambda_{n}, n>4$ follow their respective pseudo-fixed point values on all scales in the perturbative approach, one should keep in mind that the cutoff is sent to infinity in this case. In an asymptotically free theory $\lambda_{6}$ would vanish in the far ultraviolet and correspond to a UV-repulsive direction. Therefore, to stay on an asymptotically free renormalization group trajectory would require a fine-tuned value of $\lambda_{6}$ in the infrared, such that one would lie exactly on the UV-critical surface. This corresponds to setting $\lambda_{6}$ essentially equal to the running fixed point value. Because the Standard Model is not asymptotically free, there is no reason to require that $\lambda_{6}$ should be set to its running pseudo-fixed point value in the ultraviolet. Toward the infrared, it will still approach the same value. In an effective theory $\lambda_{6}$ can be chosen independently of the pseudo-fixed point at $\Lambda$. A given ultraviolet completion will actually dictate the ultraviolet boundary condition. A similar statement applies to all higherdimensional couplings.

\section{Models for high-scale physics}

Starting with an effective field theory at a finite scale $\Lambda$, the corresponding action can depend on many additional free parameters in the top-Higgs sector. An example is given by $\lambda_{6}$ as studied in section 2 . These parameters can have a sizeable influence on the stability of the Higgs potential at high energy scales. In section 2.4 we demonstrated that the interplay of just the $\lambda_{6}$ term with the Higgs quartic coupling shifts the limits on the measured Higgs mass from naive stability arguments by several per-cent. Let us now address the key question as to whether such an effect can be generated in a particle physics model, by integrating out heavy states. Depending on the choice of cutoff scale, we would of course expect that the microscopic model could also contain gravitational degrees of freedom. Here, we will focus on a much simpler toy model to demonstrate the presence of higher-order couplings at $\Lambda$.

\subsection{Heavy scalars}

As a first step, let us check if additional heavy scalars can provide a model for new physics above $\Lambda$, which features stable potentials with $\lambda_{6}(\Lambda)>0$ and $\lambda_{4}(\Lambda)<0$. In the simplest scenario, the cutoff scale $\Lambda$ corresponds to the mass scale of additional states, which are coupled to the Standard Model. For scales $k>\Lambda$ they contribute to the renormalization group flow of the SM couplings shown in eq. (2.7). To compute the allowed Higgs masses in such a scenario, we do not need to consider a UV-complete theory for the heavy states beyond $\Lambda$. Instead, the model can come with an inherent cutoff, $\Lambda_{\mathrm{BSM}} \gg \Lambda$. This corresponds to a hierarchy of effective theories, in which the Standard Model is superseded by a model containing heavy scalars, which again will be embedded in a more fundamental model close to the Planck scale. 
In our simple model we couple the additional scalar to the Standard Model through a Higgs portal [72-91] added to the effective potential of eq. (2.6),

$$
\Delta V_{\mathrm{eff}}(k)=\lambda_{H S}(k) \frac{H^{2}}{2} \frac{S^{2}}{2}+m_{S}(k)^{2} \frac{S^{2}}{2}+\lambda_{S}(k) \frac{S^{4}}{4} .
$$

Due to the reflection symmetry $S \rightarrow-S$, the heavy scalar is stable and could constitute (a part of) the dark matter relic density. We assume that this $\mathbb{Z}_{2}$ symmetry remains unbroken. The additional massive scalar field adds new loop terms to the beta functions for $\lambda_{4}$ and $\lambda_{6}$, contributing only for $k>m_{S}$. To decouple the massive modes below $m_{S}$ we include threshold terms of the form $1 /\left(1+m_{S}^{2} / k^{2}\right)^{n}$ with an appropriate power $n$. Including these threshold effects, the loop terms can be calculated in the FRG scheme, as shown in appendix C. If we allow for $N_{S}$ mass-degenerate scalar fields with the same Higgs portal interaction, the one-loop beta functions of our toy model, eq. (2.7), become

$$
\begin{aligned}
& \beta_{\lambda_{4}}=\frac{1}{8 \pi^{2}}\left[-n_{y} N_{c} y^{4}+2 n_{y} N_{c} y^{2} \lambda_{4}+9 \lambda_{4}^{2}-\frac{15}{4} \lambda_{6}+c_{\lambda} g_{F}^{4}+\frac{N_{S} \lambda_{H S}^{2}}{4\left(1+m_{S}^{2} / k^{2}\right)^{3}}\right] \\
& \beta_{\lambda_{6}}=2 \lambda_{6}+\frac{1}{16 \pi^{2}}\left[2 n_{y} N_{c} y^{6}+6 n_{y} N_{c} y^{2} \lambda_{6}-108 \lambda_{4}^{3}+90 \lambda_{4} \lambda_{6}-\frac{N_{S} \lambda_{H S}^{3}}{2\left(1+m_{S}^{2} / k^{2}\right)^{4}}\right] .
\end{aligned}
$$

The Higgs portal contributions to the beta functions follow the usual pattern for bosonic fluctuations: $\beta_{\lambda_{4}}$ receives a positive contribution, while for $\beta_{\lambda_{6}}$ the sign is reversed, etc. Most relevant to our argument is the negative sign in $\beta_{\lambda_{6}}$, because it implies a growing coupling toward the infrared, and thus a stabilizing effect. Looking at the effective potential we can generalize the stabilizing effect of heavy scalars beyond the leading terms in a polynomial expansion, namely

$$
\frac{d}{d \log k} \frac{V_{\mathrm{eff}}(k)}{k^{4}} \sim \frac{1}{1+\lambda_{H S} H^{2}} .
$$

Through the overall positive sign heavy scalars indeed decrease the value of the effective potential toward the infrared. Furthermore, the strength of the effect depends on the value of the Higgs field, and is largest at small field values of $H$. In the following, we will restrict ourselves to $\lambda_{4}$ and $\lambda_{6}$, but keep in mind that higher-dimensional couplings will be generated with alternating signs. While truncating this series at finite order could suggest either stability or instability, the contribution to the effective potential generated by heavy scalars remains stable, and features a minimum at vanishing field.

For approximately constant $\lambda_{H S}(k)$ the value of $\lambda_{4}(\Lambda)$ will depend on $-\log \left(\frac{\Lambda_{\mathrm{BSM}}}{\Lambda}\right)$. Thus, the bare value $\lambda_{4}\left(\Lambda_{\mathrm{BSM}}\right)$ will determine $\lambda_{4}(\Lambda)$. On the other hand, higherdimensional couplings such as $\lambda_{6}$ reach values which are independent of $\Lambda_{\mathrm{BSM}}$, as expected from their canonical dimensionality: as in the case of the pure Standard Model, the infrared value of $\lambda_{6}$ in the presence of a heavy scalar is determined by a pseudo-fixed point. In figure 8 we demonstrate that the behavior of $\lambda_{6}$ is completely determined in terms of this strongly IR-attractive pseudo-fixed point, making the value of $\lambda_{6}\left(\Lambda_{\mathrm{BSM}}\right)$ irrelevant for weak-scale observables, as long as $\Lambda \ll \Lambda_{\mathrm{BSM}}$. All three scenarios shown yield the correct values $m_{h}=125 \mathrm{GeV}$ and $m_{t}^{\text {(pole) }}=173 \mathrm{GeV}$. The value of $\lambda_{6}(\Lambda)$ only depends on the 

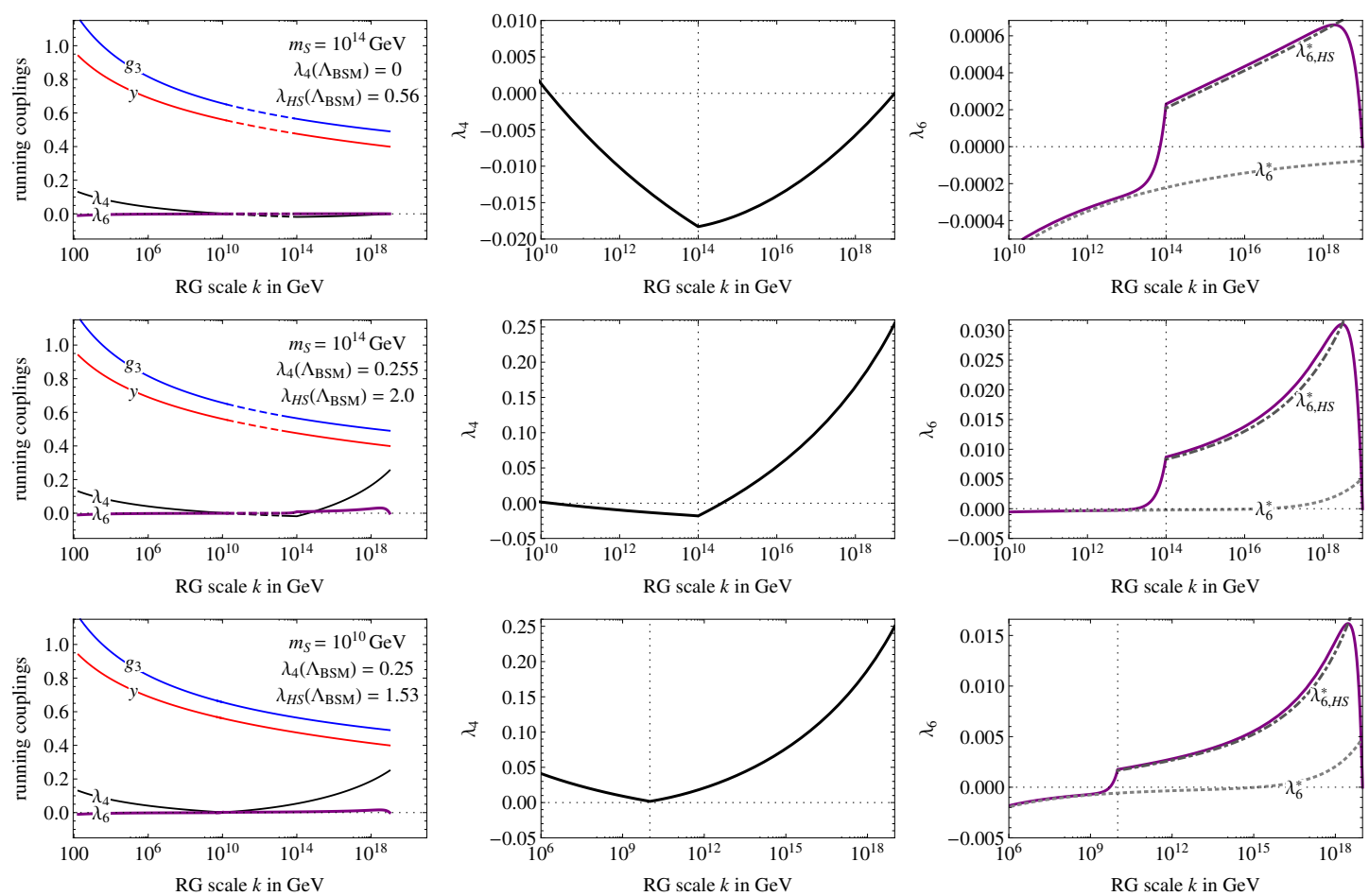

Figure 8. Running couplings in the presence of a Higgs portal coupling $\lambda_{H S}$ and $N_{S}=3$. In the top row the boundary condition is $\lambda_{4}\left(\Lambda_{\mathrm{BSM}}\right)=0$, while in the second row it is replaced by a finite value. In the bottom row we illustrate a moderately big $\lambda_{H S} \sim 1$ at the Planck scale, which is sufficient for an absolutely stable potential at all scales. In the right panels, we show the pseudo fixed point value for $\lambda_{6}$ in the presence of heavy scalars, $\lambda_{6, H S}^{*}$, and without heavy scalars, $\lambda_{6}^{*}$.

relevant and marginal couplings at that scale. As expected from universality arguments, $\lambda_{6}$ forgets the dynamics between $\Lambda_{\mathrm{BSM}}$ and $\Lambda$, different from the marginally relevant coupling $\lambda_{4}$, which does depend on the details of the dynamics at $\Lambda_{\mathrm{BSM}}$.

Comparing the full solution to the pseudo-fixed point trajectory in and beyond the Standard Model we indeed observe that setting $\lambda_{6}(\Lambda)=\lambda_{6 \mathrm{SM}}^{*}$ (and correspondingly for all higher-dimensional operators) is too restrictive. Instead, the value of $\lambda_{6}$ is determined by an interplay of two pseudo-fixed points: as long as $k>\Lambda, \lambda_{6}$ is determined by the pseudo-fixed point in the presence of new physics. Below the scale of new physics, $\lambda_{6}$ undergoes a transition to the pseudo-fixed point determined by the relevant and marginal Standard Model couplings. A caveat is that this analysis applies only to situations with small anomalous dimensions, i.e. in a perturbative regime.

We can now determine the conditions under which $\lambda_{4}(\Lambda)$ and $\lambda_{6}(\Lambda)$ assume values that according to section 2.4 yield Higgs masses below the conventional lower bound. First, we re-iterate our earlier observation that seeming instabilities in the potential in figure 8 are artifacts of our truncation to $\lambda_{4}$ and $\lambda_{6}$. To fix $\lambda_{4}$ and $\lambda_{6}$ we set $\lambda_{4}\left(\Lambda_{\mathrm{BSM}}\right)$ such that the Higgs mass comes out correctly. We then solve the pseudo-fixed point equation for $\lambda_{6}$, which determines its value at $\Lambda$. We obtain

$$
\lambda_{6}(\Lambda)=\frac{-12 y^{6}(\Lambda)+216 \lambda_{4}^{3}(\Lambda)+N_{S} \lambda_{H S}^{3}(\Lambda)}{4\left(16 \pi^{2}+9 y^{2}(\Lambda)+45 \lambda_{4}(\Lambda)\right)} .
$$


A positive top Yukawa and a negative Higgs quartic coupling, which is the case of interest for the Higgs mass bounds, each reduce the pseudo-fixed point value. As the top Yukawa coupling grows toward the infrared, this negative contribution increases. Accordingly, a sufficiently large and positive value of $\lambda_{H S}$ is needed for a positive fixed-point value $\lambda_{6}^{*}$, which is then depleted toward lower scales. In contrast, $\lambda_{4}$ is driven to increasingly negative values by the fluctuations of the heavy scalar. This implies that while the generated value of $\lambda_{6}$ provides a potential that is bounded from below, it is not always large enough to yield a potential with a minimum at vanishing field values. The size of the quadratic Higgs term $\mu^{2}$ still decides whether the potential at the cutoff scale $\Lambda$ is meta-stable, or features a global minimum at vanishing Higgs field.

It is nevertheless possible to generate initial conditions corresponding to a stable bare potential with a minimum at a vanishing field value. As an example, we use $\Lambda=10^{14} \mathrm{GeV}$, where $y(\Lambda)=0.476$ and $\lambda_{4}(\Lambda)=-0.017$ give a physical Higgs mass of $125 \mathrm{GeV}$. This value of $\lambda_{4}(\Lambda)$ can be reached by adjusting $\lambda_{4}\left(\Lambda_{\mathrm{BSM}}\right)$. To obtain a UV-stable potential with the measured Higgs mass value, we need

$$
N_{S} \lambda_{H S}^{3} \gtrsim 24
$$

for example corresponding to $\lambda_{H S} \sim 2$ and 3 additional scalars. This large value of $N_{S} \lambda_{H S}^{3}$ is needed to compensate for the factor 216 in front of the $\lambda_{4}$ term in eq. (3.4), which arises from the combinatorics of the respective scalar diagrams. Accordingly, a new physics scenario in which the new states are not combinatorically disfavored in comparison to the Higgs can accommodate larger values of $\lambda_{6}$ without needing large numbers of new states and/or large couplings.

In our example, the large value of the Higgs portal coupling implies that the heavy scalar sector will be driven toward a Landau pole not far above $\Lambda_{\mathrm{BSM}}$. In such simple models one could therefore conclude that a UV-stable potential for the Higgs sector can be generated at the cost of a non-perturbative regime not far above $\Lambda_{\mathrm{BSM}}$.

\subsection{Heavy fermions}

An obvious open question is the effect of heavy fermions. If their mass is generated through symmetry breaking at the electroweak scale, they will have a large Yukawa coupling. For example models featuring a heavy chiral fourth generation show a significantly reduced value of the possible cutoff scale $\Lambda$ since the extra fermions would just add to the problematic effect of the top quark. Furthermore, such models are experimentally excluded through the Higgs coupling measurements at the LHC.

We consider a model where the additional fermions are heavy, but their Yukawa coupling is small. In this setting we rely on an unspecified symmetry breaking mechanism at a high scale, affecting only the additional fermions. In our simple model we include such a mass term without discussing its possible origin. While this is straightforward in the present $\mathbb{Z}_{2}$ model, in spite of the mass term explicitly breaking the $\mathbb{Z}_{2}$ symmetry, an embedding of such a mechanism in the Standard Model with its $\mathrm{SU}(2)_{L}$ gauge invariance is less clear. The relevant Lagrangian terms for $N_{\eta}$ heavy fermions then take the form

$$
\mathcal{L}_{\eta} \supset y_{\eta} H \bar{\eta} \eta+m_{\eta}^{2} \bar{\eta} \eta
$$


Alternatively, we could consider a model where the additional fermions are singlets under the Standard Model gauge groups. In that case they can have a mass even in the unbroken phase. Their Higgs couplings would correspond to a dimension-5 operator of the form $H^{2} \bar{\eta} \eta$.

Let us again analyze the induced potential at $\Lambda=m_{\eta}$ in terms of the pseudo-fixed point for $\lambda_{6}$, which instead of eq. (3.4) now gets the additional contribution

$$
\lambda_{6}(\Lambda)=\frac{-12 y^{6}(\Lambda)+216 \lambda_{4}^{3}(\Lambda)+N_{S} \lambda_{H S}^{3}(\Lambda)-12 N_{\eta} y_{\eta}^{6}(\Lambda)}{4\left(16 \pi^{2}+9 y^{2}(\Lambda)+45 \lambda_{4}(\Lambda)+9 N_{\eta} y_{\eta}^{2}(\Lambda)\right)} .
$$

The fermion $\eta$ induces a negative contribution to $\lambda_{6}$. In general, its contribution to $\lambda_{n}$ will be positive for $n / 2$ even, and negative for $n / 2$ odd. This means that heavy fermions will generically make it hard to reach sizeable positive values of $\lambda_{6}$, but they might be of interest for cases where, e.g., $\lambda_{8}>0$ stabilizes the potential.

Alternatively, heavy fermions can be important in a scale invariant theory in the far ultraviolet, $V\left(\Lambda_{\mathrm{BSM}}\right)=0$. Here, fermions help to control the induced value of $\lambda_{4}(\Lambda)$. In a simple analysis the masses of heavy bosons and fermions split the new physics scale $\Lambda$ into two thresholds $m_{S}=\Lambda_{S}$ and $m_{\eta}=\Lambda_{\eta}$, where we assume $m_{S}<m_{\eta}$. The induced value of $\lambda_{4}$ for $\Lambda_{S}<\Lambda_{\eta}$ now reads

$$
\lambda_{4}\left(\Lambda_{S}\right)=\lambda_{4}\left(\Lambda_{\mathrm{BSM}}\right)+\left(\frac{3}{8 \pi^{2}} y^{4}\left(\Lambda_{S}\right)-\frac{N_{S}}{32 \pi^{2}} \lambda_{H S}\left(\Lambda_{S}\right)^{2}\right) \log \frac{\Lambda_{\mathrm{BSM}}}{\Lambda_{S}}+\frac{N_{\eta}}{8 \pi^{2}} y_{\eta}^{4}\left(\Lambda_{\eta}\right) \log \frac{\Lambda_{\mathrm{BSM}}}{\Lambda_{\eta}},
$$

where we assume a slow running in the Yukawa and the portal couplings to the heavy scalars. A hierarchy $m_{\eta}>m_{S}$ implies that the value of the induced coupling $\lambda_{6}$ will be independent of $y_{\eta}$ and instead be determined by the pseudo-fixed point at $\Lambda_{S}$. In this framework we can construct models where $\lambda_{4}$ is small and negative and $\lambda_{6}=\mathcal{O}(1)$, without the need for large fermion and boson numbers or large couplings.

\subsection{Non-perturbative new physics}

In the last two sections we have shown that simple models with additional heavy fermions and bosons allow us to generate initial conditions for the renormalization group flow which yield low Higgs masses based on a UV-stable potential. The main challenge is to avoid the non-perturbative regime beyond $\Lambda_{\mathrm{BSM}}$ while generating coupling values $\left|\lambda_{6}\right|>\left|\lambda_{4}\right|$. In general, the effect of new physics on the beta functions of the running couplings in the Higgs sector follows two different patterns:

1. terms independent of the Higgs self-couplings induce these couplings, even if they vanish at some scale $\Lambda_{\mathrm{BSM}}$;

2. terms which change the scaling of the Higgs self-couplings by inducing an anomalous dimension $\eta_{\mathrm{NP}}$. 
This means we can write the beta functions for $\lambda_{4}$ and $\lambda_{6}$ in the presence of new heavy states as

$$
\begin{aligned}
& \beta_{\lambda_{4}}=\frac{1}{8 \pi^{2}}\left[-n_{y} N_{c} y^{4}+2 n_{y} N_{c} y^{2} \lambda_{4}+9 \lambda_{4}^{2}-\frac{15}{4} \lambda_{6}+c_{\lambda} g_{F}^{4}+c_{4 \mathrm{NP}}\right]+\eta_{4 \mathrm{NP}} \lambda_{4} \\
& \beta_{\lambda_{6}}=2 \lambda_{6}+\frac{1}{16 \pi^{2}}\left[2 n_{y} N_{c} y^{6}+6 n_{y} N_{c} y^{2} \lambda_{6}-108 \lambda_{4}^{3}+90 \lambda_{4} \lambda_{6}+c_{6 \mathrm{NP}}\right]+\eta_{6 \mathrm{NP}} \lambda_{6},
\end{aligned}
$$

where $c_{4 / 6 \mathrm{NP}}$ and $\eta_{4 / 6 \mathrm{NP}}$ encode the effects of the microscopic degrees of freedom beyond the Standard Model. Again, it is useful to analyze the running of $\lambda_{6}$ in terms of a pseudofixed point of the kind shown in eq. (2.21),

$$
\begin{aligned}
\lambda_{6 *}=-\frac{c}{2+\eta} \quad \text { with } \quad c & =\frac{2 n_{y} N_{c} y^{6}-108 \lambda_{4}^{3}+c_{6 \mathrm{NP}}}{16 \pi^{2}} \\
\eta & =\frac{6 n_{y} N_{c} y^{2}+90 \lambda_{4}}{16 \pi^{2}}+\eta_{6 \mathrm{NP}} .
\end{aligned}
$$

For example, we can start with vanishing values for all couplings at $\Lambda_{\mathrm{BSM}}$. We can induce a negative value of $\lambda_{4}$ with increasing magnitude and a positive value of $\lambda_{6}$, if we choose $c_{4 \mathrm{NP}}>0$ and $c_{6 \mathrm{NP}}<0$ and $\left|c_{6 \mathrm{NP}}\right|>2 n_{y} N_{c} y^{6}$. The pseudo-fixed point moves toward smaller values as $\lambda_{4}$ grows in magnitude toward the infrared. As long as $\eta_{6} \mathrm{NP}$ is small the pseudo-fixed point stays strongly infrared attractive.

Let us now broaden our approach and allow for new physics in a non-perturbative regime, such that large anomalous dimensions of either sign can exist. An example could be asymptotic safety [108-110]. We choose a scenario where $\eta_{6 \mathrm{NP}} \simeq-2$, i.e., the scaling dimension of the higher-dimensional coupling changes from irrelevant to marginal, or even relevant. In such a case, the pseudo-fixed point will not be strongly infrared attractive anymore. Accordingly, new physics can induce a sizeable value of $\lambda_{6}$, that is not depleted between $\Lambda_{\mathrm{BSM}}$ and $\Lambda$. From eq. (3.10) it is clear that cancelling the canonical dimensionality by a large anomalous dimension can lead to significantly enhanced values for $\lambda_{6}^{*}$.

As an example, we set $c_{4 \mathrm{NP}}=9 / 4, c_{6 \mathrm{NP}}=-27 / 2, \eta_{4 \mathrm{NP}}=0$, and $\eta_{6 \mathrm{NP}}=-2$ and assume that the effect of new physics appears beyond $\Lambda=10^{14} \mathrm{GeV}$. We then obtain $\lambda_{4}(\Lambda)=-0.026$ and $\lambda_{6}(\Lambda)=0.51$, as shown in figure 9 . This does provide a UV-stable potential with Higgs masses significantly below the conventional lower bound.

\section{Conclusions}

With the discovery of the Higgs boson the Standard Model describing the interactions between fundamental states in terms of a gauge theory is complete. The crucial question becomes whether the Standard Model itself gives us a hint where new physics must appear [111]. One possible clue is the behavior of the Higgs potential at high energy scales and large field values. A perturbative analysis of the Higgs potential in the Standard Model indicates that it loses its stability around $10^{10} \mathrm{GeV}$, well below the Planck scale. We have investigated the robustness of this claim in the presence of higher-dimensional operators in the effective Higgs potential, as one would expect in theories with a physical UV-cutoff. Because of their canonical dimension the effects of these higher-dimensional operators will 


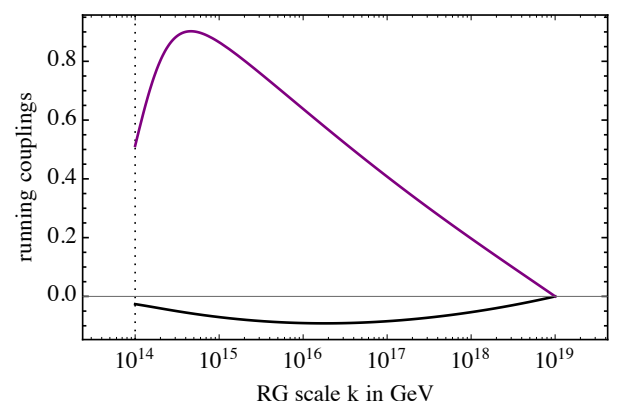

Figure 9. We show $\lambda_{4}$ (black) and $\lambda_{6}$ (purple), obtained using the model beta functions from eq. (3.9), which include new-physics effects beyond $\Lambda=10^{14} \mathrm{GeV}$ (dotted vertical line), induce these couplings and alter their canonical scaling dimensionality.

vanish quickly toward the infrared, but nevertheless can play a crucial role in the vicinity of the cutoff. We identify several scenarios:

- The UV-potential including higher-dimensional couplings with $\lambda_{4}<0$ is stable, as is the effective potential. The electroweak minimum remains the global minimum throughout the RG evolution. In this case, already the $H^{6}$ operator allows us to extend the UV-cutoff by about two orders of magnitude. Further higher-dimensional operators may increase the cutoff scale further.

- The UV-potential and the effective potential are stable with their respective global electroweak minima. However, during the RG evolution this minimum seems only meta-stable for an intermediate range of scales. For conclusive statements about this pseudo-stable scenario, a more complete treatment of the RG evolution of the effective potential is necessary. We expect that part of this parameter space belongs to the class of fully stable scenarios whereas other parts may feature a real metastability. Taking the scenario literally, the barrier separating the two minima of the $k$-dependent potential is typically of the order $k$.

- The UV-potential is meta-stable, featuring a global minimum at some high field value. This can be achieved for an arbitrarily small initial $\lambda_{6}$, and therefore no instability occurs even for arbitrarily large negative $\lambda_{4}$. Implementing the constraint that the electroweak minimum has to be sufficiently long-lived, this essentially reduces to the standard discussion of a viably long-lived meta-stable region.

Higher-dimensional operators affect the constraints on the Higgs mass between $\sim$ $1 \mathrm{GeV}$ for the stable potential to $\sim 4 \mathrm{GeV}$ for a pseudo-stable setup. In the presence of an ultraviolet cutoff, the size of higher-dimensional couplings is an inherent ambiguity of the Standard Model. To include higher-dimensional operators in our setup we have used non-perturbative functional renormalization group techniques in the presence of a finite UV-cutoff. Neglecting higher-dimensional operators, our approach and the usual perturbative approach agree in the domain where perturbation theory with a massless regularization scheme is applicable, i.e., for energies and field values much smaller than the UV-cutoff 
$\Lambda$. With regard to higher-dimensional operators, perturbation theory assumes very specific and small values, essentially those generated by the Standard Model degrees of freedom and encoded in higher-order effects. Our non-perturbative treatment allows for more general values of the higher-dimensional couplings.

The difference between the values of the couplings in perturbation theory and our more general values is in principle measurable. These effects become sizeable at energies where the new dimensionless couplings are of order one. Restricting the higher-dimensional couplings to be of order one at the cutoff scale (where they typically have their largest values), limits the size of the achievable shifts, but even in the absence of higher-dimensional operators, stability up to the Planck scale is only disfavored by $\sim 3 \sigma$ [45]. Small shifts as observed in our analysis can significantly reduce this tension.

Physics beyond the Standard Model predicts the size of the Higgs self-interactions at the cutoff. We can ask what type of new physics generates higher-dimensional operators of suitable size, such that Higgs masses below the conventional lower bound can be obtained. In general, UV-completions featuring large anomalous dimensions can easily generate sizeable higher-dimensional operators, stabilizing the Higgs potential up to the Planck scale. Their effects can be linked directly to the more general analysis in terms of higher-dimensional operators contributing to the Higgs potential.

In weakly coupled models the answer is less obvious. Our approach to new physics affecting vacuum stability is different from the usual strategy of modifying the running of the Higgs potential through new particles below $10^{10} \mathrm{GeV}$, as discussed in the appendix. In the main body of our paper we focus on very heavy new particles, coupled to the Standard Model through renormalizable operators. They modify the Higgs potential through induced higher-dimensional Higgs self-couplings. Specifically, we have investigated a simple extension of the Standard Model by heavy scalars and fermions. While it is possible to generate sizeable higher-dimensional operators, the required parameter choices in our simple model (couplings and number of fields) are on the border of being non-perturbative. It remains an intriguing question, what kind of new physics can generate higher-dimensional operators of a suitable size, while remaining perturbative on all scales.

\section{Acknowledgments}

We thank Christof Wetterich and Jan Martin Pawlowski for discussions. TP would like to thank Christof Wetterich for giving him the friendly-supportive Christof smile when he heard about this project. The work of AE is supported by an Imperial College Junior Research Fellowship. This research was supported in part by Perimeter Institute for Theoretical Physics. Research at Perimeter Institute is supported by the Government of Canada through Industry Canada and by the Province of Ontario through the Ministry of Research and Innovation. AE and RS thank the ITP at Heidelberg University, and MMS the Perimeter Institute for hospitality during the course of this work. MMS is supported by the grant ERC-AdG-290623. HG and RS acknowledge support by the DFG under grants GRK1523, Gi328/5-2 (Heisenberg program). 


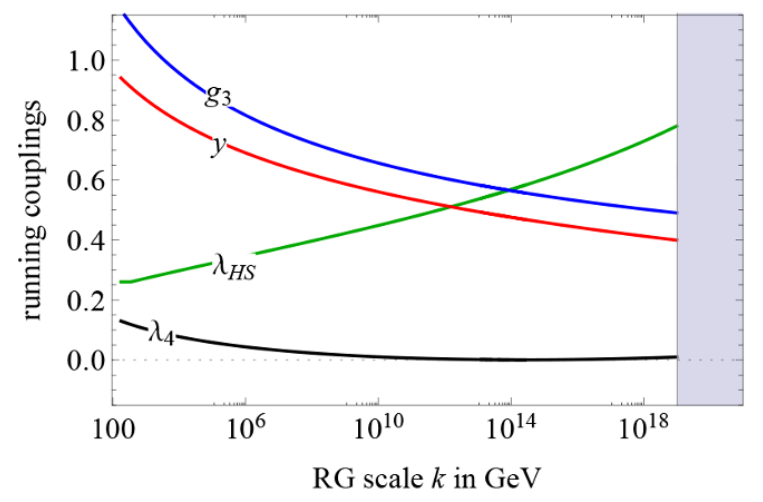

Figure 10. We show the running of the couplings employing eq. (2.7), amended by an additional Higgs portal coupling $\lambda_{H S}$.

\section{A Light dark matter scalars}

Different scenarios predicting new particles which modify the relation $m_{H}(\Lambda)$ from the conventional lower bound can be distinguished by the ratio of their mass to the cutoff $\Lambda$. In the main body of this paper we focus on heavy states with mass $m \gtrsim \Lambda$. Their fluctuations induce an effective potential $V_{\text {eff }}(\Lambda)$ which generically contains non-vanishing higher-dimensional terms.

A different scenario arises using light states with mass $m \gtrsim v$. Their primary effect is to modify the running of (some of) the Standard Model couplings between the electroweak scale and the cutoff scale. Most importantly, they can alter the RG flow of the quartic Higgs self-coupling, such that it remains positive all the way to the cutoff $\Lambda$. One example is a gauge singlet, dark matter scalar. It is coupled to the Standard Model through the Higgs portal defined in eq. (3.1), $\lambda_{H S} H^{2} S^{2} / 4$, but now with a mass $m_{S}$ in the GeV-TeV region [49, 72-91]. With a $\mathbb{Z}_{2}$ symmetry protecting it from decaying and in the absence of a second VEV which would lead to mixing with the Higgs scalar, the only additional parameters of this model are $m_{S}$ and $\lambda_{H S}$. This makes it the arguably simplest model, which can accommodate the complete observed dark matter relic density [92]. From a field theory point of view, the portal coupling cannot be large and negative to avoid an unstable combined scalar potential. To yield the desired effect on the Higgs mass bound it should be positive.

The difference to the additional heavy scalar discussed in section 3.1 is that $\lambda_{H S}$ is now important at scales $k<\Lambda$. A sufficiently large $\lambda_{H S}$ can altogether prevent $\lambda_{4}$ from becoming negative. In figure 10 we update results from ref. [49] by including the effect of the strong gauge coupling and approximating the effect of the weak couplings through a fiducial contribution as defined in eq. (2.7). A moderately large $\lambda_{H S} \sim 0.78$ at the Planck scale is indeed sufficient for an absolutely stable potential for the Higgs at all scales. In our updated analysis we also guarantee the correct weak-scale masses $m_{h}=125 \mathrm{GeV}$ and

$m_{t}^{\text {(pole) }}=173 \mathrm{GeV}$. The infrared value of $\lambda_{H S}$ in figure 10 is compatible with a dark matter mass of $m_{S}=340 \mathrm{GeV}$, if we require the new scalar to constitute the complete dark matter relic density [92]. 
From this example we tentatively conclude that the positive contribution of $\lambda_{H S}$ to $\beta_{\lambda_{4}}$ is sufficient to reconcile the measured value of the Higgs mass with a stable Higgs potential on all scales up to the Planck scale. As a crucial phenomenological distinction to our investigation in the main part of this paper, new physics states appear at low scales $m_{S} \gtrsim v$, instead of at mass scales $m_{S} \sim \Lambda$. Thus the new light states can become experimentally accessible in dark matter searches of at colliders in the near future. The particular region of parameter space used in figure 10 will be probed by planned upgrades of XENON [93, 94] and LUX [95, 96].

\section{B Tunnelling}

In this appendix we briefly discuss estimates for the tunneling rates in the presence of a $\sim \lambda_{6} \varphi^{6}$-term [64-67]. The tunnelling rate can be calculated by determining the Euclidean action of bubbles $S_{\mathrm{E}}\left[\varphi_{\text {bounce }}\right]$, where $\varphi_{\text {bounce }}$ is the bounce solution of the Euclidean equations of motion for a real scalar field

$$
\frac{d^{2} \varphi_{\text {bounce }}}{d r^{2}}+\frac{3}{r} \frac{d \varphi_{\text {bounce }}}{d r}-V^{\prime}\left(\varphi_{\text {bounce }}\right)=0
$$

with the boundary conditions $\varphi_{\text {bounce }}(\infty)=\varphi_{\text {bounce }}=\varphi_{+}$and $\varphi_{\text {bounce }}^{\prime}(0)=0$. The value $\varphi_{+}$is the location of the meta-stable minimum. The tunneling rate then becomes

$$
\frac{\Gamma}{V} \sim e^{-S_{\mathrm{E}}\left[\varphi_{\text {bounce }}\right]} .
$$

Let us now turn to the potential given by eq. (2.6), namely

$$
V(\varphi)=\frac{m_{\varphi}^{2}}{2} \varphi^{2}+\frac{\lambda_{4}}{4} \varphi^{4}+\frac{\lambda_{6}}{8 k^{2}} \varphi^{6}
$$

For the formation of a Bubble of size $\sim R$ one should evaluate the scale dependent potential at the scale $k \sim 1 / R[97,98]$. In the situation we are interested in, the field values where the potential starts to be lower than the electroweak vacuum near zero vev are quite large. Accordingly we are interested in very small bubbles and $k \gtrsim 10^{10} \mathrm{GeV}$. Neglecting higherorder terms, the potential would be unstable with $m_{\varphi}^{2}>0$ and $\lambda_{4}<0$. To stabilize the potential we can add a $\lambda_{6} \varphi^{6}$ term with, $\lambda_{6}>0$.

We are mainly interested in an upper limit on the tunneling rate. It therefore makes sense to approximate the potential eq. (B.3) by a potential which is always lower than the potential we actually have. A simple choice is to simply take the term

$$
V_{\text {approx }}(\varphi)=\frac{1}{4} \lambda_{4} \varphi^{4} \leq V(\varphi) .
$$

For such a potential the tunneling rate has been computed in ref. [98]. In this case the bounce solution is,

$$
\begin{aligned}
\varphi_{\text {bounce }} & =\sqrt{\frac{2}{\left|\lambda_{4}\right|}} \frac{2 R}{r^{2}+R^{2}} \\
S_{\mathrm{E}}\left[\varphi_{\text {bounce }}\right] & =\frac{8 \pi^{2}}{3\left|\lambda_{4}\right|} .
\end{aligned}
$$


Because the approximate potential is scale invariant we actually have infinitely many bounces corresponding to different values of $R$, and in principle we have to take all of them into account.

To determine the pre-factor of the exponential one has to perform a one-loop calculation around the background of the bounce solution [99]. Doing this [98] one finds for the tunnelling probability in a space time volume $V_{4}$

$$
p \sim \frac{V_{4}}{R^{4}} \exp \left(-\frac{8 \pi^{2}}{3\left|\lambda_{4}(1 / R)\right|}\right) .
$$

The scale dependence of $\lambda_{4}$ breaks the scale invariance and differently sized bubbles have different actions. To determine the loop-correction one has to calculate the inverse of the determinant over the spectrum of the fluctuation modes around the bounce background. The zero-modes have to be treated with some extra care. They correspond to integrations over the corresponding collective coordinates. The volume factor arises from the translation zero-modes and the corresponding integration over the space-time volume. The scale invariance of the approximate potential leads to an additional zero-mode in the spectrum. The integral over $d R / R$ is the integration over the collective coordinate corresponding to this zero-mode [100]. Taking all possible bubbles into account we finally arrive at

$$
p \sim \int \frac{d R}{R} \frac{V_{4}}{R^{4}} \exp \left(-\frac{8 \pi^{2}}{3\left|\lambda_{4}(1 / R)\right|}\right) .
$$

For constant $\lambda_{4}(1 / R)$ this integral is divergent for small values of $R$. Such small values of $R$ correspond to a large maximal field values of the bounce solution, as can be seen from eq. (B.5). Indeed we have,

$$
\varphi_{\text {bounce }}(r=0) \sim \frac{1}{R} .
$$

This tells us how the integral is regularized in presence of $\lambda_{6}>0$ which stabilizes the potential. For field values beyond the point where the potential has its minimum and starts to rise again, the approximation of the potential by just the $-\lambda_{4} \varphi^{4} / 4$ term is not sensible anymore and we should cut off the integral at this point at the latest.

In practice, the integral is typically dominated by a reasonably sized region around the most negative values of $\lambda_{4}$ because of the strong exponential suppression of the integrand for small $\left|\lambda_{4}\right|$ in eq. (B.7). Even a small upturn in $\lambda_{4}(1 / R)$ typically quickly overcomes any growth in the $1 / R^{4}$ factor. Indeed a better approximation is probably to use an effective quartic coupling

$$
\lambda_{4}^{\mathrm{eff}}=\lambda_{4}+\frac{\lambda_{6}}{2 k^{2}} \varphi^{2}
$$

Here, one can again see the stabilizing effect of a positive $\lambda_{6}$. With increasing field value, i.e. decreasing $R$ of the bounce, $\lambda_{4}^{\text {eff }}$ becomes less negative effectively cutting off the integral. More intuitively, an increasing $\lambda_{6}$ lowers the depth of the minimum, and thus lowers the tunneling probability.

Let us now determine the value of the meta-stability bound on $\lambda_{4}$. For the space-time volume we can take the Hubble volume and time,

$$
V_{4} \sim \frac{1}{H_{0}^{4}}
$$


where $H_{0}$ is today's value of the Hubble constant. Longevity of the vacuum requires that the tunneling probability in the volume $V_{4} \sim 1 / H_{0}^{4}$ is smaller than 1 . For $R$ between $R=$ $1 /\left(10^{10} \mathrm{GeV}\right)$ and $R=1 /\left(10^{19} \mathrm{GeV}\right)$ this corresponds to a bounce action $S_{\mathrm{E}} \gtrsim 400-500$. Therefore, as long as

$$
\left|\lambda_{4}\right| \leq 0.052
$$

we are on the safe side. In the presence of higher-dimensional operators, this inequality will be relaxed, and thus represents a very conservative upper limit.

A similar argument can be made when the higher dimensional operator is actually destabilizing, i.e., $\lambda_{6}<0$. In this a suitable approximate potential is

$$
V_{\text {approx }}(\varphi)=\frac{\lambda_{4}^{\min }}{4} \varphi^{4}, \quad \text { with } \quad \lambda_{4}^{\min }=\lambda_{4}+\frac{\lambda_{6}}{2 k^{2}} \varphi_{\max }^{2}
$$

where $\varphi_{\max }$ is the value where the potential is once again stabilized by even higher dimensional terms. Using the same arguments as above the limit becomes

$$
\left|\lambda_{4}^{\min }\right|=\left|\lambda_{4}+\frac{\lambda_{6}}{2 k^{2}} \varphi_{\max }^{2}\right| \leq 0.052
$$

We conclude, in accordance with refs. [64-66] that the presence of higher-order operators could have a significant impact on the lifetime of the electroweak vacuum in a meta-stable situation, for example if $\lambda_{6}$ is negative. Estimates relying solely on $\lambda_{4}$ strictly only apply to a restricted set of UV-completions. Large higher dimensional operators, in particular ones that destabilize the potential can have significant effects.

\section{Functional renormalization group}

The $\beta$-functions for the running couplings used in this work follow naturally from the functional renormalization group (RG). Formulated in terms of a flow equation for a momentum-scale-dependent effective action $\Gamma_{k}$, the functional RG provides for efficient means to follow the flow of higher-dimensional operators, to account for threshold effects and to even approach regimes of strong coupling. Of course, the universal one-loop $\beta$-functions dominantly used in the main text follow as a simple by-product in the weakcoupling limit.

In the following, we briefly recall the essentials of the functional RG, concentrating on its application to the present model. As this method has already been employed in the present context to the pure $\mathbb{Z}_{2}$ Higgs-Yukawa model [54], we focus on the generalizations for the SU(3) gauged version. Finally, we detail how the universal one-loop $\beta$-functions arise by neglecting higher-loop terms as well as RG improvement and by considering the 'deep Euclidean region'.

The functional RG is a manifestation of the Wilsonian idea of solving a quantum field theory by integrating out fluctuations in the path integral, momentum shell by momentum shell. In its modern version, it can be conveniently formulated in terms of a scale-dependent action functional $\Gamma_{k}$. This is constructed such that it interpolates between the UV-theory parameterized at the cutoff scale $\Lambda$ in terms of the $\Gamma_{k \rightarrow \Lambda} \rightarrow S_{\Lambda}$ and the full effective action 
in the IR, $\Gamma_{k \rightarrow 0} \rightarrow \Gamma_{\text {eff. }}$ The latter contains, for instance, the full effective potential as a local term without derivatives of the fields, $\Gamma_{\text {eff }}=\int d^{4} x\left(V_{\text {eff }}+\mathcal{O}\left((\partial \phi)^{2}\right)\right)$. The change of the effective action as a function of the RG scale $k$ ( $\mathrm{RG}$ flow) is governed by the Wetterich equation [60]

$$
\partial_{t} \Gamma_{k}=\frac{1}{2} \mathrm{~S} \operatorname{Tr}\left[\frac{\partial_{t} R_{k}}{\Gamma_{k}^{(2)}+R_{k}}\right], \quad t=\ln \frac{k}{\Lambda},
$$

where $\Gamma_{k}^{(2)}$ denotes the second derivative of $\Gamma_{k}$ with respect to the fluctuating fields $(H$, $\left.\psi, \bar{\psi}, A_{\mu}, \cdots\right)$; the super-trace includes a minus sign for fermionic trace parts. It also involves a summation over the eigenvalues of the regularized propagator, $\left(\Gamma^{(2)}+R_{k}\right)^{-1}$, which in our case reduces to a momentum integration. A new technical ingredient is given by the regulator $R_{k}$ which can be thought of as a momentum-dependent and $k$-dependent mass term. Specifying $R_{k}$ corresponds to specifying the details of the momentum shells regularization. Different choices of $R_{k}$ correspond to different regularization schemes.

Even though the Wetterich equation has a simple one-loop structure, it is an exact equation as there exists an exact equivalence to the full functional integral. The key ingredient for this is that the propagator in the loop $\sim\left(\Gamma_{k}^{(2)}+R_{k}\right)$ denotes the full propagator at the scale $k$. From the full solution of eq. (C.1), for instance, all correlation functions can be computed.

While a perturbative expansion of the Wetterich equation reproduces perturbation theory to any order, the flow equation can also be used to extract non-perturbative information [112-116]. Following the Wilsonian idea, the scale dependent action $\Gamma_{k}$ contains not just relevant or marginal couplings, but encodes effects of high-scale quantum fluctuations in the presence of higher-order operators. For practical calculations, this infinite series of operators in the effective action is truncated. In the present context, a useful ordering scheme defining our approximation of the exact flow is given by an expansion of the effective action in terms of derivatives of the field. To next-to-leading order this gives

$$
\Gamma_{k}=\int d^{4} x\left[V+\frac{Z_{H}}{2}\left(\partial_{\mu} H\right)^{2}+\sum_{j=1}^{n_{f}} Z_{\psi_{j}} \bar{\psi}_{j} i \not D \psi_{j}+i \frac{1}{\sqrt{2}} \sum_{j=1}^{n_{y}} \bar{y}_{j} H \bar{\psi}_{j} \psi_{j}+\frac{Z_{G}}{4} F_{\mu \nu}^{a} F^{a \mu \nu}\right]
$$

where the potential $V$, the (bare) Yukawa couplings $\bar{y}_{j}$ as well as all wave function renormalizations $Z_{H, \psi, G}$ are $k$-dependent. The gauge-part of the action is also supplemented by a gauge-fixing and Fadeev-Popov ghost contribution.

Inserting this ansatz into the Wetterich equation and expanding both sides in terms of this basis of operators leads to the $\beta$-functions, i.e. the flow equations for the Yukawa couplings and the wave function renormalizations. For the flow of the effective potential, we obtain a $\beta$-functional defining a $\beta$-function for every field value $H$. For clarity, let us write down this flow of the effective potential explicitly:

$$
\beta_{V}(H)=\frac{d V(H)}{d \log k}=\frac{k^{4}}{32 \pi^{2}}\left[\frac{1-\frac{\eta_{H}}{6}}{1+\frac{V^{\prime \prime}(H)}{k^{2} Z_{H}}}-4 \sum_{j=1}^{n_{y}} N_{\mathrm{c}} \frac{1-\frac{\eta_{\psi_{j}}}{5}}{1+\frac{\bar{y}_{j}^{2} H^{2}}{2 k^{2} Z_{\psi_{j}}^{2}}}\right] .
$$


Here, we have introduced the anomalous dimensions of the fluctuation fields, defined by the wave function renormalization flow,

$$
\eta_{H}=-\frac{d \log Z_{H}}{d \log k}, \quad \eta_{\psi_{j}}=-\frac{d \log Z_{\psi_{j}}}{d \log k}, \quad \eta_{G}=-\frac{d \log Z_{G}}{d \log k} .
$$

In eq. (C.3), we have also made an explicit choice for the regulator function in terms of the linear regulator [117]. Other choices lead to a qualitatively similar behavior on the right hand side. This expression clearly contains threshold effects, where for example the scalar contribution will be dampened once the scalar mass, related to $V^{\prime \prime}$, becomes of order $k$.

From eq. (C.3), the $\beta$-functions for the scalar self-interactions $\lambda_{2 n}$ can be obtained straightforwardly. To do this, we expand the potential in powers of the field,

$$
V_{\mathrm{eff}}(k)=\frac{\bar{\mu}(k)^{2}}{2} H^{2}+\sum_{n=2} \frac{\bar{\lambda}_{2 n}(k)}{k^{2 n-4}}\left(\frac{H^{2}}{2}\right)^{n}
$$

and introduce the renormalized couplings,

$$
\mu^{2}=\frac{\bar{\mu}^{2}}{Z_{H}}, \quad \lambda_{2 n}=\frac{\bar{\lambda}_{2 n}}{Z_{H}^{n}}, \quad y=\frac{\bar{y}_{j}}{Z_{\psi_{j}} Z_{H}^{1 / 2}}, \quad g_{s}^{2}=\frac{\bar{g}_{s}^{2}}{Z_{G}} .
$$

Here, the rescaling with the wave function renormalizations compensate for the field rescalings which are necessary to bring eq. (C.2) to a canonical form, e.g., $H \rightarrow H / Z_{H}^{1 / 2}$, etc. Inserting the expansion of eq. (C.5) into eq. (C.3), and expanding both sides to fourth order in the field, we can read off the flow of $\lambda_{4}$,

$$
\beta_{\lambda_{4}}=2 \eta_{H} \lambda_{4}+\frac{1}{16 \pi^{2}}\left[9 \lambda_{4}^{2} \frac{1-\frac{\eta_{H}}{6}}{\left(1+\frac{\mu^{2}}{k^{2}}\right)^{3}}-\frac{15}{2} \lambda_{6} \frac{1-\frac{\eta_{H}}{6}}{\left(1+\frac{\mu^{2}}{k^{2}}\right)^{2}}\right]-\frac{1}{8 \pi^{2}} \sum_{j=1}^{n_{y}} N_{\mathrm{c}} y^{4}\left(1-\frac{\eta_{\psi_{j}}}{5}\right)
$$

We observe that the scalar loop terms $\sim \lambda_{4}^{2}$ and $\sim \lambda_{6}$ are suppressed if the mass parameter is larger than the RG scale $k$. This signals a typical threshold effect characterizing the decoupling of massive modes. In the present application, however, $\mu^{2}$ is chosen such that it remains small for all values of $k$ and eventually drops below zero near the weak scale $k_{\mathrm{EW}}$. Then all modes become massive and decouple, which can be made explicit in the flow by expanding about $H=v$.

For the high-energy applications discussed in the main text, we take the limit of the deep Euclidean region, $\mu^{2} / k^{2} \rightarrow 0$, as is standard in perturbative computations. Using the one-loop result for the anomalous dimension $\eta_{H}$ (also obtained from the flow equation by studying the flow of the scalar kinetic term), $\eta_{H}=\sum_{j=1}^{n_{y}} N_{\mathrm{c}} y_{j}^{2} /(4 \pi)^{2}$, we obtain the $\beta_{\lambda_{4}}$ function as given in eq. (2.7), except for the last term $\sim g_{F}^{4}$ modelling the electroweak gauge sector which is described in the main text. In fact, the ansatz of eq. (C.2) also yields higherorder terms like $\sim \lambda_{4}^{2} \eta_{H}$, corresponding to contributions from higher loops. The fact that these terms arise from the one-loop formula in eq. (C.1) is due to the 'RG-improvement' of the propagator in the loop. 
For the perturbative estimates discussed in the main text, these higher-loop contributions are neglected, cf. eq. (2.7), whereas they are fully included in the FRG results of figure 6 further outlined below.

Similarly, $\beta_{\lambda_{6}}$ of eq. (2.7) is obtained from an expansion of eq. (C.3) to order $H^{6}$, taking the deep-Euclidean limit and ignoring RG-improvement terms. Also a contribution $\sim \lambda_{8}$ is ignored in eq. (2.7), whereas it is included in the FRG flow studies, see below. The derivation of $\beta_{y}$ of eq. (2.7) with $y_{j} \rightarrow y$ for $n_{y}=1$ follows the same pattern. In the general case, the flow of the Yukawa couplings for different fermions can be derived in this way; then, the different fermions also acquire separate anomalous dimensions. The standard one-loop $\beta_{g_{s}}$ function for the gauge sector can most easily be derived from the flow equation, using the background-gauge formalism. Here, the running of $g_{s}$ is directly related to the anomalous dimension, $\beta_{g_{s}}=\eta_{G} g_{s} / 2$ see e.g. [112-116].

The manner by which we extracted the one-loop $\beta$-functions from the Wetterich equation follows a general pattern: first expand both sides in terms of the operators under consideration; this yields the generally non-perturbative $\beta$-function of the corresponding coupling within a given ansatz. For the one-loop order, all RG-improvement terms corresponding to higher-loop corrections can simply be dropped. The universal one-loop coefficients arise in the limit of the deep Euclidean region which is the expected necessary prerequisite for one-loop universality. While our explicit examples discussed above have used the linear regulator as a special choice, it can be proved that the one-loop coefficients for the renormalizable operators are manifestly scheme independent. However, the $\beta$-function coefficients for $\lambda_{6}$ do depend on the choice of the regulator. This scheme dependence is physical in the sense that it parameterizes how the Standard Model as an effective theory is embedded into an underlying theory.

For the non-perturbative functional $\mathrm{RG}$ results in the main text we integrate the $\beta$ functions for the full ansatz eq. (C.2), including the mass parameter $\mu^{2}(k)$, all threshold dependences and all RG-improvement terms due to the back-feeding of anomalous dimensions. For the flow of the effective potential we use a polynomial expansion to order $H^{8}$. Higher orders can easily be included, but do not lead to any significant change of results. For the pure $\mathbb{Z}_{2}$ model, the local convergence has been checked to order $\sim \lambda_{40} H^{40}$ [54].

The flow equations for the pure $\mathbb{Z}_{2}$ model are given in refs. [54, 118] for a general regulator function and the linear regulator. To match them to the present matter content, all fermion loop contributions have to be multiplied by a factor $\sum_{j=1}^{n_{y}} N_{c}$, corresponding to the number of fermions interacting with the scalar sector. In the following, we confine ourselves to listing only the new contributions induced by the $\mathrm{SU}\left(N_{c}\right)$ gauge sector. The electroweak sector will be modelled in terms of a fiducial coupling, as in eq. (2.7).

Whereas the form of the flow of the effective potential in eq. (C.3) or eq. (12) in ref. [54] remains unaffected, the flow of the Yukawa coupling receives direct contributions from the gauge sector. Also for numerical purposes, it is convenient to write this $\beta$-function as

$$
\begin{array}{r}
\frac{d y_{j}^{2}}{d \log k}=2 \times \text { eq. (13) of [54]+ } \frac{(3+\xi)}{4 \pi^{2}} \frac{N_{c}^{2}-1}{2 N_{c}} g_{s}^{2} y_{j}^{2}\left[2 \kappa y_{j}^{2} l_{2,1}^{(F B) 4}\left(\kappa y_{j}^{2}, 0 ; \eta_{\psi_{j}}, \eta_{G}\right)\right. \\
\left.-l_{1,1}^{(F B) 4}\left(\kappa y_{j}^{2}, 0 ; \eta_{\psi_{j}}, \eta_{G}\right)\right] .
\end{array}
$$




\begin{tabular}{|l|ccc|}
\hline & $\lambda_{4}$ & $y$ & $g_{s}$ \\
\hline$k=m_{t}^{(\text {pole })}$ & 0.129 & 0.940 & 1.167 \\
$k=1 \mathrm{TeV}$ & 0.101 & 0.867 & 1.060 \\
\hline
\end{tabular}

Table 1. Values of the model parameters at the top pole mass and the RG matching scale $k=1 \mathrm{TeV}$ for the matching between the perturbative RG and the functional RG trajectories.

The factor of 2 in front of the first term is due to different conventions for the Yukawa coupling. The dictionary for the conventions used in this work and in ref. [54] reads: $y_{j}^{2} \hat{=} 2 h^{2}, H^{2} / Z_{H} \hat{=} 2 k^{2} \tilde{\rho}, V(H) \hat{=} k^{4} u(\tilde{\rho})$, where the l.h.s. corresponds to this work and the r.h.s. to [54]. The dimensionless quantity $\kappa$ is related to the expansion point of the potential in field space; in the present work, we have $\kappa=0$ at high-energy scales and $\kappa=v^{2} /\left(2 k^{2}\right)$ in the broken regime near the weak scale. The threshold functions $l$ (and $m$ below) describe the (regulator-dependent) decoupling of massive modes; for any admissible regulator, they approach finite constants for vanishing first arguments and decrease to zero for large first arguments. The result holds for arbitrary Lorenz-gauge parameter $\xi$. All numerical studies have been performed in the Landau gauge $\xi=0$.

Correspondingly, the fermion anomalous dimension reads

$$
\begin{aligned}
\eta_{\psi_{j}}=\text { eq. (16) of }[54]+\frac{1}{16 \pi^{2}} \frac{N_{c}^{2}-1}{2 N_{c}} g_{s}^{2}[ & (3-\xi) m_{1,2}^{(F B) d}\left(\kappa y_{j}^{2}, 0 ; \eta_{G}\right) \\
& \left.-3(1-\xi) \tilde{m}_{1,1}^{(F B) d}\left(\kappa y_{j}^{2}, 0 ; \eta_{G}\right)\right],
\end{aligned}
$$

while the corresponding equation for the scalar anomalous dimension $\eta_{H}$ remains form invariant to eq. (15) of ref. [54].

In practice, the resulting system of coupled differential equations is solved numerically. For stability reasons in the presence of higher-dimensional operator, the flow is solved from the UV down to the weak scale. The boundary conditions in the UV are chosen implicitly such that the infrared observables are matched as described below.

\section{Mapping between flow trajectories}

To study the RG evolution of the Standard Model, its physical masses and couplings have to be related to the fundamental parameters appearing in the $\beta$-functions. Within the perturbative setting this procedure is well-established up to NNLO [45]. For our perturbative results we follow these prescriptions at the corresponding loop order, setting the parameters at the universal $\mathrm{RG}$ scale $k=m_{t}^{\text {(pole) }}$ such that they reproduce a running top mass $m_{t}=164 \mathrm{GeV}$, a running Higgs mass $m_{h}=125 \mathrm{GeV}$, and the strong coupling $\alpha_{S}\left(m_{Z}\right)=0.1184$. We list the explicit numbers employed in our calculations for our model with $n_{y}=1$ in table 1 .

The functional RG approach allows us to access the regime of spontaneously broken symmetry, where a non-vanishing minimum $H_{\min , k} \neq 0$ of the effective potential appears in the RG flow. This way all particle masses are generated dynamically. In contrast to the perturbative setting, the running minimum enters the RG evolution of the Higgs 

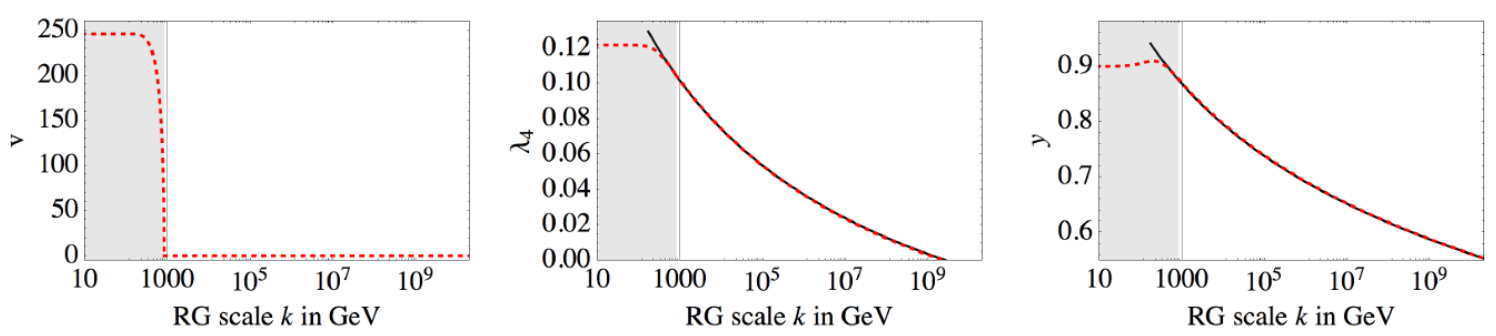

Figure 11. Comparison and matching of FRG flows with the perturbative setting. The left panel distinguishes the symmetric regime and the regime of spontaneously broken symmetry. The center and right panels show the FRG flows (red dotted) as well as the perturbative flows (black curve) of the Higgs coupling and the top Yukawa coupling. The running couplings are matched at $1 \mathrm{TeV}$.

quartic coupling and the Yukawa coupling explicitly: the generated running masses $m_{t}(k) \propto$ $y H_{\min , k}$ and $m_{h}(k) \propto \sqrt{\lambda_{4}} H_{\min , k}$ induce a threshold behavior that leads to a freeze-out of model runnings in the infrared, as shown in figure 11. The FRG flow for the RG relevant direction parameterized by the mass parameter $\mu^{2}$ has to be fine-tuned at the UV-cutoff scale, such that the minimum of the effective potential approaches the SM value of the vev in the infrared $H_{\min , k \rightarrow 0}=v$. This fine-tuning is a manifestation of the hierarchy problem, which is dealt with here by an explicit choice of UV-parameters.

The threshold behavior in the FRG is non-universal, which means it depends on the choice of regulator function. This dependence is natural as it parametrizes the details of decoupling of massive modes from the flow. In the symmetry-broken regime, the threshold behavior leads to a significant deviation of the running couplings from their values in the perturbative setting, which is strictly tied to the symmetric deep Euclidean region; the latter is, of course, inappropriate for the threshold region. To compare to the perturbative results and extract universal physical results, we have to establish a matching procedure. We choose to match the flows at the scale $1 \mathrm{TeV}$, which is sufficiently deep inside the Euclidean region but not yet affected by possible higher-dimensional operators, cf. table 1 .

Our procedure equates the running couplings in the perturbative $\overline{\mathrm{MS}}$ scheme and the FRG scheme at the matching scale. Approaching that scale from the ultraviolet, typical FRG trajectories are still in the symmetric regime, an electroweak minimum of the potential has not been generated yet, and the threshold effects in the FRG $\beta$-functions are subleading. In figure 11 we see that this regime features flows of the Higgs self-coupling and the top Yukawa coupling closely resembling the perturbative RG flows. As the values for the couplings agree with those of the $\overline{\mathrm{MS}}$ scheme at the matching scale, our matching scheme guarantees that UV-initial conditions for the FRG flow are mapped to physical values in the infrared with high accuracy. Qualitatively, this mapping works already at the level of our FRG trajectories without a matching procedure, since threshold effects automatically provide for a freeze-out of the RG flow, such that in principle physical values can be extracted at $k=0$. In a more elaborate FRG setup, using a more sophisticated truncation of the operator space, our mapping using $\overline{\mathrm{MS}}$ parameters will become obsolete and quantitatively precise values for physical observables can be read off from the FRG flow trajectories at $k=0$ directly. 


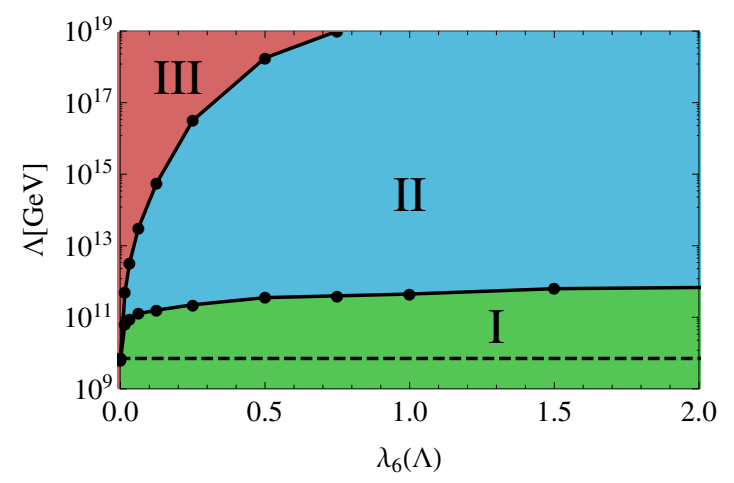

Figure 12. Different stability regions as a function of $\Lambda$ and $\lambda_{6}$ analogous to figure 6 for the simple model. In region I (green) the potential is stable everywhere; in region II (blue) the UVpotential is stable, while the potential is only pseudo-stable for intermediate scales $v<k<\Lambda$; in region III (red) the UV-potential violates the unique-minimum condition. The inclusion of electroweak contributions to the full potential leads only to minor modifications of the border between region I and II. Region III becomes somewhat larger as the smaller mass parameter $\mu^{2}$ facilitaes a violation of the unique-minimum condition already for smaller cutoffs.

Our choice of matching scale is also applicable when the symmetry-breaking scale lies at around $1 \mathrm{TeV}$, as threshold effects remain small close to that scale. Matching the flows at $1 \mathrm{TeV}$ therefore provides us with a well-defined procedure to compare the two RG schemes. As an added benefit we can thoroughly study the FRG evolution of our toy model consistently including higher-dimensional operators and threshold effects in a Standard Model context.

\section{E Modelling electroweak contributions to the full effective potential}

In the present work, the electroweak contributions are modelled by means of a fiducial gauge coupling contributing to the perturbative $\beta$-functions eq. (2.7). In particular, we neglected a similar contribution to the flow of $\lambda_{6}$ in this simple model. With the full $\beta$-functional for the full effective potential eq. (C.3) at hand, we can in fact model the electroweak gauge contributions to the full potential including the flow of the mass-parameter $\mu^{2}(k)$ as well as all higher couplings $\lambda_{6,8, \ldots}(k)$. To lowest order, we add the contribution of a fiducial gauge loop to the $\beta$-functional [107],

$$
\text { eq. }(\text { C.3 }) \rightarrow \text { eq. (C.3) }+\frac{k^{4}}{32 \pi^{2}} \frac{c_{V}}{1+\frac{g_{F}^{2} H^{2}}{4 k^{2}}},
$$

with the fiducial gauge coupling $g_{F}$ and a numerical constant $c_{V}$. As in the main text, we choose these fiducial parameters in such a way that the running of the quartic coupling $\lambda_{4}$ is quantitatively similar to the running of the Standard Model Higgs quartic coupling, cf. eq. (2.7) and figure 1. Comparing to eq. (2.7), we identify $c_{V}=16 c_{\lambda}=9$. This choice leads to the same flow equation for the quartic coupling as in the main text. In addition, also the flow equations of all other couplings in the scalar sector $\left(\mu^{2}(k), \lambda_{6,8, \ldots}(k)\right)$ receive contributions proportional to an appropriate power of the fiducial gauge coupling $g_{F}$. The flow 
of all scalar couplings is only slightly modified such that the solution for the simple model lies almost on top of the results with the advanced fiducial modification eq. (E.1). The only relevant quantitative change is the flow of the mass parameter $\mu^{2}(k)$. The additional bosonic degrees of freedom reduce the ratio $\mu^{2}(k) / k^{2}$ on a wide range of scales.

The main modification introduced by the advanced model eq. (E.1) affects the validity regime of the unique-minimum condition eq. (2.14). This leads to a shift of the border between region II and III of figure 6 . Smaller values for $\mu^{2}(\Lambda)$ have to be compensated by a smaller UV cutoff $\Lambda$ for fixed $\lambda_{4}(\Lambda)$ and $\lambda_{6}(\Lambda)$, resulting in a depletion of the transition curve. This effect is less pronounced at the transition between region I and II because the relative reduction in $\mu^{2} / k^{2}$ is smaller.

Open Access. This article is distributed under the terms of the Creative Commons Attribution License (CC-BY 4.0), which permits any use, distribution and reproduction in any medium, provided the original author(s) and source are credited.

\section{References}

[1] P.W. Higgs, Broken symmetries, massless particles and gauge fields, Phys. Lett. 12 (1964) 132 [INSPIRE].

[2] P.W. Higgs, Broken Symmetries and the Masses of Gauge Bosons, Phys. Rev. Lett. 13 (1964) 508 [INSPIRE].

[3] P.W. Higgs, Spontaneous Symmetry Breakdown without Massless Bosons, Phys. Rev. 145 (1966) 1156 [INSPIRE].

[4] F. Englert and R. Brout, Broken Symmetry and the Mass of Gauge Vector Mesons, Phys. Rev. Lett. 13 (1964) 321 [INSPIRE].

[5] G.S. Guralnik, C.R. Hagen and T.W.B. Kibble, Global Conservation Laws and Massless Particles, Phys. Rev. Lett. 13 (1964) 585 [inSPIRE].

[6] ATLAS collaboration, Observation of a new particle in the search for the Standard Model Higgs boson with the ATLAS detector at the LHC, Phys. Lett. B 716 (2012) 1 [arXiv: 1207.7214] [INSPIRE].

[7] CMS collaboration, Observation of a new boson at a mass of $125 \mathrm{GeV}$ with the CMS experiment at the LHC, Phys. Lett. B 716 (2012) 30 [arXiv:1207.7235] [INSPIRE].

[8] L. Maiani, G. Parisi and R. Petronzio, Bounds on the Number and Masses of Quarks and Leptons, Nucl. Phys. B 136 (1978) 115 [INSPIRE].

[9] N. Cabibbo, L. Maiani, G. Parisi and R. Petronzio, Bounds on the Fermions and Higgs Boson Masses in Grand Unified Theories, Nucl. Phys. B 158 (1979) 295 [inSPIRE].

[10] R.F. Dashen and H. Neuberger, How to Get an Upper Bound on the Higgs Mass, Phys. Rev. Lett. 50 (1983) 1897 [INSPIRE].

[11] D.J.E. Callaway, Nontriviality of Gauge Theories With Elementary Scalars and Upper Bounds on Higgs Masses, Nucl. Phys. B 233 (1984) 189 [InSPIRE].

[12] M.A.B. Beg, C. Panagiotakopoulos and A. Sirlin, Mass of the Higgs Boson in the Canonical Realization of the Weinberg-Salam Theory, Phys. Rev. Lett. 52 (1984) 883 [INSPIRE]. 
[13] M. Lindner, Implications of Triviality for the Standard Model, Z. Phys. C 31 (1986) 295 [INSPIRE].

[14] J. Kuti, L. Lin and Y. Shen, Upper Bound on the Higgs Mass in the Standard Model, Phys. Rev. Lett. 61 (1988) 678 [INSPIRE].

[15] T. Hambye and K. Riesselmann, Matching conditions and Higgs mass upper bounds revisited, Phys. Rev. D 55 (1997) 7255 [hep-ph/9610272] [INSPIRE].

[16] I.V. Krive and A.D. Linde, On the Vacuum Stability Problem in Gauge Theories, Nucl. Phys. B 117 (1976) 265 [INSPIRE].

[17] N.V. Krasnikov, Restriction of the Fermion Mass in Gauge Theories of Weak and Electromagnetic Interactions, Yad. Fiz. 28 (1978) 549 [INSPIRE].

[18] P.Q. Hung, Vacuum Instability and New Constraints on Fermion Masses, Phys. Rev. Lett. 42 (1979) 873 [INSPIRE].

[19] H.D. Politzer and S. Wolfram, Bounds on Particle Masses in the Weinberg-Salam Model, Phys. Lett. B 82 (1979) 242 [Erratum ibid. 83B (1979) 421] [InSPIRE].

[20] A.D. Linde, Vacuum Instability, Cosmology and Constraints on Particle Masses in the Weinberg-Salam Model, Phys. Lett. B 92 (1980) 119 [INSPIRE].

[21] M. Lindner, M. Sher and H.W. Zaglauer, Probing Vacuum Stability Bounds at the Fermilab Collider, Phys. Lett. B 228 (1989) 139 [INSPIRE].

[22] C. Ford, D.R.T. Jones, P.W. Stephenson and M.B. Einhorn, The Effective potential and the renormalization group, Nucl. Phys. B 395 (1993) 17 [hep-lat/9210033] [INSPIRE].

[23] G. Altarelli and G. Isidori, Lower limit on the Higgs mass in the standard model: An update, Phys. Lett. B 337 (1994) 141 [INSPIRE].

[24] T. Plehn, Lectures on LHC Physics, Lect. Notes Phys. 844 (2012) 1 [arXiv:0910.4182] [INSPIRE].

[25] P.B. Arnold, Can the Electroweak Vacuum Be Unstable?, Phys. Rev. D 40 (1989) 613 [INSPIRE].

[26] M. Sher, Precise vacuum stability bound in the standard model, Phys. Lett. B 317 (1993) 159 [Addendum ibid. B 331 (1994) 448] [hep-ph/9307342] [INSPIRE].

[27] M. Sher, Electroweak Higgs Potentials and Vacuum Stability, Phys. Rept. 179 (1989) 273 [INSPIRE].

[28] P.B. Arnold and S. Vokos, Instability of hot electroweak theory: bounds on $m(H)$ and $M(t)$, Phys. Rev. D 44 (1991) 3620 [inSPIRE].

[29] J.R. Espinosa and M. Quirós, Improved metastability bounds on the standard model Higgs mass, Phys. Lett. B 353 (1995) 257 [hep-ph/9504241] [INSPIRE].

[30] J.A. Casas, J.R. Espinosa and M. Quirós, Improved Higgs mass stability bound in the standard model and implications for supersymmetry, Phys. Lett. B 342 (1995) 171 [hep-ph/9409458] [INSPIRE].

[31] J.A. Casas, J.R. Espinosa and M. Quirós, Standard model stability bounds for new physics within LHC reach, Phys. Lett. B 382 (1996) 374 [hep-ph/9603227] [INSPIRE].

[32] B. Bergerhoff, M. Lindner and M. Weiser, Dynamics of metastable vacua in the early universe, Phys. Lett. B 469 (1999) 61 [hep-ph/9909261] [INSPIRE]. 
[33] G. Isidori, G. Ridolfi and A. Strumia, On the metastability of the standard model vacuum, Nucl. Phys. B 609 (2001) 387 [hep-ph/0104016] [INSPIRE].

[34] N. Arkani-Hamed, S. Dubovsky, L. Senatore and G. Villadoro, (No) Eternal Inflation and Precision Higgs Physics, JHEP 03 (2008) 075 [arXiv:0801.2399] [inSPIRE].

[35] J. Ellis, J.R. Espinosa, G.F. Giudice, A. Hoecker and A. Riotto, The Probable Fate of the Standard Model, Phys. Lett. B 679 (2009) 369 [arXiv:0906.0954] [INSPIRE].

[36] J. Elias-Miro, J.R. Espinosa, G.F. Giudice, G. Isidori, A. Riotto and A. Strumia, Higgs mass implications on the stability of the electroweak vacuum, Phys. Lett. B 709 (2012) 222 [arXiv: 1112.3022] [INSPIRE].

[37] O. Lebedev, On Stability of the Electroweak Vacuum and the Higgs Portal, Eur. Phys. J. C 72 (2012) 2058 [arXiv:1203.0156] [INSPIRE].

[38] J. Elias-Miro, J.R. Espinosa, G.F. Giudice, H.M. Lee and A. Strumia, Stabilization of the Electroweak Vacuum by a Scalar Threshold Effect, JHEP 06 (2012) 031 [arXiv:1203.0237] [INSPIRE].

[39] G. Degrassi et al., Higgs mass and vacuum stability in the Standard Model at NNLO, JHEP 08 (2012) 098 [arXiv: 1205.6497] [INSPIRE].

[40] S. Alekhin, A. Djouadi and S. Moch, The top quark and Higgs boson masses and the stability of the electroweak vacuum, Phys. Lett. B 716 (2012) 214 [arXiv:1207.0980] [INSPIRE].

[41] L.A. Anchordoqui et al., Vacuum Stability of Standard Model ${ }^{++}$, JHEP 02 (2013) 074 [arXiv:1208.2821] [INSPIRE].

[42] I. Masina, Higgs boson and top quark masses as tests of electroweak vacuum stability, Phys. Rev. D 87 (2013) 053001 [arXiv:1209.0393] [INSPIRE].

[43] F. Bezrukov and M. Shaposhnikov, Why should we care about the top quark Yukawa coupling?, arXiv:1411.1923 [INSPIRE].

[44] F. Bezrukov, M.Y. Kalmykov, B.A. Kniehl and M. Shaposhnikov, Higgs Boson Mass and New Physics, JHEP 10 (2012) 140 [arXiv:1205.2893] [INSPIRE].

[45] D. Buttazzo et al., Investigating the near-criticality of the Higgs boson, JHEP 12 (2013) 089 [arXiv: 1307.3536] [INSPIRE].

[46] M. Shaposhnikov and C. Wetterich, Asymptotic safety of gravity and the Higgs boson mass, Phys. Lett. B 683 (2010) 196 [arXiv: 0912.0208] [InSPIRE].

[47] M. Holthausen, K.S. Lim and M. Lindner, Planck scale Boundary Conditions and the Higgs Mass, JHEP 02 (2012) 037 [arXiv:1112.2415] [INSPIRE].

[48] A. Hebecker, A.K. Knochel and T. Weigand, The Higgs mass from a String-Theoretic Perspective, Nucl. Phys. B 874 (2013) 1 [arXiv: 1304.2767] [InSPIRE].

[49] A. Eichhorn and M.M. Scherer, Planck scale, Higgs mass and scalar dark matter, Phys. Rev. D 90 (2014) 025023 [arXiv: 1404.5962] [INSPIRE].

[50] W. Altmannshofer, W.A. Bardeen, M. Bauer, M. Carena and J.D. Lykken, Light Dark Matter, Naturalness and the Radiative Origin of the Electroweak Scale, JHEP 01 (2015) 032 [arXiv: 1408.3429] [INSPIRE].

[51] Z. Fodor, K. Holland, J. Kuti, D. Nogradi and C. Schroeder, New Higgs physics from the lattice, PoS(LATTICE 2007)056 [arXiv:0710.3151] [INSPIRE]. 
[52] P. Hegde, K. Jansen, C.-J.D. Lin and A. Nagy, Stabilizing the electroweak vacuum by higher dimensional operators in a Higgs-Yukawa model, PoS (LATTICE 2013) 058 [arXiv:1310.6260] [INSPIRE].

[53] D.Y.J. Chu, K. Jansen, B. Knippschild, C.J.D. Lin, K.-I. Nagai and A. Nagy, Phase structure and Higgs boson mass in a Higgs-Yukawa model with a dimension-6 operator, arXiv: 1501.00306 [INSPIRE].

[54] H. Gies, C. Gneiting and R. Sondenheimer, Higgs Mass Bounds from Renormalization Flow for a simple Yukawa model, Phys. Rev. D 89 (2014) 045012 [arXiv:1308.5075] [InSPIRE].

[55] H. Gies and R. Sondenheimer, Higgs Mass Bounds from Renormalization Flow for a Higgs-top-bottom model, Eur. Phys. J. C 75 (2015) 68 [arXiv:1407.8124] [INSPIRE].

[56] V. Branchina and H. Faivre, Effective potential (in)stability and lower bounds on the scalar (Higgs) mass, Phys. Rev. D 72 (2005) 065017 [hep-th/0503188] [InSPIRE].

[57] V. Branchina, H. Faivre and V. Pangon, Effective potential and vacuum stability, J. Phys. G 36 (2009) 015006 [arXiv:0802 .4423] [InSPIRE].

[58] C.P. Burgess, V. Di Clemente and J.R. Espinosa, Effective operators and vacuum instability as heralds of new physics, JHEP 01 (2002) 041 [hep-ph/0201160] [INSPIRE].

[59] W. Rodejohann and H. Zhang, Impact of massive neutrinos on the Higgs self-coupling and electroweak vacuum stability, JHEP 06 (2012) 022 [arXiv:1203.3825] [INSPIRE].

[60] C. Wetterich, Exact evolution equation for the effective potential, Phys. Lett. B 301 (1993) 90 [INSPIRE].

[61] M. Harada, Y. Kikukawa, T. Kugo and H. Nakano, Nontriviality of gauge Higgs-Yukawa system and renormalizability of gauged NJLS model, Prog. Theor. Phys. 92 (1994) 1161 [hep-ph/9407398] [INSPIRE].

[62] K. Holland and J. Kuti, How light can the Higgs be?, Nucl. Phys. Proc. Suppl. 129 (2004) 765 [hep-lat/0308020] [INSPIRE].

[63] K. Holland, Triviality and the Higgs mass lower bound, Nucl. Phys. Proc. Suppl. 140 (2005) 155 [hep-lat/0409112] [INSPIRE].

[64] V. Branchina and E. Messina, Stability, Higgs Boson Mass and New Physics, Phys. Rev. Lett. 111 (2013) 241801 [arXiv:1307.5193] [INSPIRE].

[65] V. Branchina, E. Messina and A. Platania, Top mass determination, Higgs inflation and vacuum stability, JHEP 09 (2014) 182 [arXiv:1407.4112] [INSPIRE].

[66] V. Branchina, E. Messina and M. Sher, Lifetime of the electroweak vacuum and sensitivity to Planck scale physics, Phys. Rev. D 91 (2015) 013003 [arXiv:1408.5302] [INSPIRE].

[67] Z. Lalak, M. Lewicki and P. Olszewski, Higher-order scalar interactions and SM vacuum stability, JHEP 05 (2014) 119 [arXiv:1402.3826] [INSPIRE].

[68] C. Wetterich, Gauge hierarchy due to strong interactions?, Phys. Lett. B 104 (1981) 269 [INSPIRE].

[69] C. Wetterich, The Mass Of The Higgs Particle, in Superstrings, unified theory and cosmology G. Furlan, J.C. Pati, D.W. Sciama, E. Sezgin and Q. Shafi eds., World Scientific, DESY-87-154 (1997). 
[70] T. Papenbrock and C. Wetterich, Two loop results from one loop computations and nonperturbative solutions of exact evolution equations, Z. Phys. C 65 (1995) 519 [hep-th/9403164] [INSPIRE].

[71] A. Codello, M. Demmel and O. Zanusso, Scheme dependence and universality in the functional renormalization group, Phys. Rev. D 90 (2014) 027701 [arXiv:1310.7625] [INSPIRE].

[72] V. Silveira and A. Zee, Scalar phantoms, Phys. Lett. B 161 (1985) 136 [INSPIRE].

[73] J. McDonald, Gauge singlet scalars as cold dark matter, Phys. Rev. D 50 (1994) 3637 [hep-ph/0702143] [INSPIRE].

[74] M.C. Bento, O. Bertolami, R. Rosenfeld and L. Teodoro, Selfinteracting dark matter and invisibly decaying Higgs, Phys. Rev. D 62 (2000) 041302 [astro-ph/0003350] [INSPIRE].

[75] C.P. Burgess, M. Pospelov and T. ter Veldhuis, The minimal model of nonbaryonic dark matter: A singlet scalar, Nucl. Phys. B 619 (2001) 709 [hep-ph/0011335] [INSPIRE].

[76] M.C. Bento, O. Bertolami and R. Rosenfeld, Cosmological constraints on an invisibly decaying Higgs boson, Phys. Lett. B 518 (2001) 276 [hep-ph/0103340] [INSPIRE].

[77] J. McDonald, Thermally generated gauge singlet scalars as selfinteracting dark matter, Phys. Rev. Lett. 88 (2002) 091304 [hep-ph/0106249] [INSPIRE].

[78] H. Davoudiasl, R. Kitano, T. Li and H. Murayama, The new minimal standard model, Phys. Lett. B 609 (2005) 117 [hep-ph/0405097] [INSPIRE].

[79] B. Patt and F. Wilczek, Higgs-field portal into hidden sectors, hep-ph/0605188 [INSPIRE].

[80] V. Barger, P. Langacker, M. McCaskey, M.J. Ramsey-Musolf and G. Shaughnessy, LHC Phenomenology of an Extended Standard Model with a Real Scalar Singlet, Phys. Rev. D 77 (2008) 035005 [arXiv:0706.4311] [INSPIRE].

[81] X.-G. He, T. Li, X.-Q. Li and H.-C. Tsai, Scalar dark matter effects in Higgs and top quark decays, Mod. Phys. Lett. A 22 (2007) 2121 [hep-ph/0701156] [INSPIRE].

[82] V. Barger, P. Langacker, M. McCaskey, M. Ramsey-Musolf and G. Shaughnessy, Complex Singlet Extension of the Standard Model, Phys. Rev. D 79 (2009) 015018 [arXiv:0811.0393] [INSPIRE].

[83] X.-G. He, T. Li, X.-Q. Li, J. Tandean and H.-C. Tsai, Constraints on Scalar Dark Matter from Direct Experimental Searches, Phys. Rev. D 79 (2009) 023521 [arXiv:0811.0658] [INSPIRE].

[84] M. Gonderinger, Y. Li, H. Patel and M.J. Ramsey-Musolf, Vacuum Stability, Perturbativity and Scalar Singlet Dark Matter, JHEP 01 (2010) 053 [arXiv:0910.3167] [INSPIRE].

[85] T.E. Clark, B. Liu, S.T. Love and T. ter Veldhuis, The Standard Model Higgs Boson-Inflaton and Dark Matter, Phys. Rev. D 80 (2009) 075019 [arXiv:0906.5595] [InSPIRE].

[86] R.N. Lerner and J. McDonald, Gauge singlet scalar as inflaton and thermal relic dark matter, Phys. Rev. D 80 (2009) 123507 [arXiv: 0909.0520] [INSPIRE].

[87] M. Kadastik, K. Kannike, A. Racioppi and M. Raidal, Implications of the $125 \mathrm{GeV}$ Higgs boson for scalar dark matter and for the CMSSM phenomenology, JHEP 05 (2012) 061 [arXiv: 1112.3647] [INSPIRE].

[88] C. Englert, T. Plehn, D. Zerwas and P.M. Zerwas, Exploring the Higgs portal, Phys. Lett. B 703 (2011) 298 [arXiv:1106.3097] [INSPIRE]. 
[89] C. Englert, T. Plehn, M. Rauch, D. Zerwas and P.M. Zerwas, LHC: Standard Higgs and Hidden Higgs, Phys. Lett. B 707 (2012) 512 [arXiv:1112.3007] [INSPIRE].

[90] M. Gonderinger, H. Lim and M.J. Ramsey-Musolf, Complex Scalar Singlet Dark Matter: Vacuum Stability and Phenomenology, Phys. Rev. D 86 (2012) 043511 [arXiv:1202.1316] [INSPIRE].

[91] C.-S. Chen and Y. Tang, Vacuum stability, neutrinos and dark matter, JHEP 04 (2012) 019 [arXiv: 1202.5717] [INSPIRE].

[92] J.M. Cline, K. Kainulainen, P. Scott and C. Weniger, Update on scalar singlet dark matter, Phys. Rev. D 88 (2013) 055025 [arXiv: 1306.4710] [inSPIRE].

[93] XENON1T collaboration, E. Aprile, The XENON1T Dark Matter Search Experiment, Springer Proc. Phys. C12-02-22 (2013) 93 [arXiv:1206.6288] [INSPIRE].

[94] XENON collaboration, P. Beltrame, Direct Dark Matter search with the XENON program, arXiv:1305.2719 [INSPIRE].

[95] LUX collaboration, S. Fiorucci, The LUX Dark Matter Search - Status Update, J. Phys. Conf. Ser. 460 (2013) 012005 [arXiv: 1301.6942] [INSPIRE].

[96] LUX collaboration, M. Woods, Underground Commissioning of LUX, arXiv:1306.0065 [INSPIRE].

[97] A. Strumia, N. Tetradis and C. Wetterich, The region of validity of homogeneous nucleation theory, Phys. Lett. B 467 (1999) 279 [hep-ph/9808263] [INSPIRE].

[98] G. Isidori, G. Ridolfi and A. Strumia, On the metastability of the standard model vacuum, Nucl. Phys. B 609 (2001) 387 [hep-ph/0104016] [INSPIRE].

[99] C.G. Callan Jr. and S.R. Coleman, The Fate of the False Vacuum. 2. First Quantum Corrections, Phys. Rev. D 16 (1977) 1762 [InSPIRE].

[100] G. 't Hooft, Computation of the Quantum Effects Due to a Four-Dimensional Pseudoparticle, Phys. Rev. D 14 (1976) 3432 [Erratum ibid. D 18 (1978) 2199] [InSPIRE].

[101] M. Sher, Electroweak Higgs Potentials and Vacuum Stability, Phys. Rept. 179 (1989) 273 [INSPIRE].

[102] G. Degrassi et al., Higgs mass and vacuum stability in the Standard Model at NNLO, JHEP 08 (2012) 098 [arXiv: 1205.6497] [INSPIRE].

[103] K.G. Chetyrkin and M.F. Zoller, Three-loop $\beta$-functions for top-Yukawa and the Higgs self-interaction in the Standard Model, JHEP 06 (2012) 033 [arXiv:1205.2892] [INSPIRE].

[104] M.J. Duncan and L.G. Jensen, Exact tunneling solutions in scalar field theory, Phys. Lett. B 291 (1992) 109 [INSPIRE].

[105] S. Moch, U. Langenfeld and P. Uwer, The Top-Quark's Running Mass, PoS (RADCOR2009) 030 [arXiv : 1001.3987] [INSPIRE].

[106] B. Schrempp and M. Wimmer, Top quark and Higgs boson masses: Interplay between infrared and ultraviolet physics, Prog. Part. Nucl. Phys. 37 (1996) 1 [hep-ph/9606386] [INSPIRE].

[107] H. Gies, S. Rechenberger, M.M. Scherer and L. Zambelli, An asymptotic safety scenario for gauged chiral Higgs-Yukawa models, Eur. Phys. J. C 73 (2013) 2652 [arXiv:1306.6508] [INSPIRE]. 
[108] S. Weinberg, Ultraviolet Divergences In Quantum Theories Of Gravitation, in General Relativity, S.W. Hawking and W. Israel, Cambridge University Press, Cambridge, U.K. (1980), pg. 790-831 [INSPIRE].

[109] M. Reuter and F. Saueressig, Quantum Einstein Gravity, New J. Phys. 14 (2012) 055022 [arXiv: 1202.2274] [INSPIRE].

[110] P. Donà, A. Eichhorn and R. Percacci, Matter matters in asymptotically safe quantum gravity, Phys. Rev. D 89 (2014) 084035 [arXiv:1311.2898] [INSPIRE].

[111] J. Jaeckel and V.V. Khoze, An upper limit on the scale of new physics phenomena from rising cross sections in high multiplicity Higgs and vector boson events, arXiv: 1411.5633 [INSPIRE].

[112] J. Berges, N. Tetradis and C. Wetterich, Nonperturbative renormalization flow in quantum field theory and statistical physics, Phys. Rept. 363 (2002) 223 [hep-ph/0005122] [INSPIRE].

[113] K. Aoki, Introduction to the nonperturbative renormalization group and its recent applications, Int. J. Mod. Phys. B 14 (2000) 1249 [InSPIRE].

[114] J.M. Pawlowski, Aspects of the functional renormalisation group, Annals Phys. 322 (2007) 2831 [hep-th/0512261] [INSPIRE].

[115] H. Gies, Introduction to the functional $R G$ and applications to gauge theories, Lect. Notes Phys. 852 (2012) 287 [hep-ph/0611146] [INSPIRE].

[116] J. Braun, Fermion Interactions and Universal Behavior in Strongly Interacting Theories, J. Phys. G 39 (2012) 033001 [arXiv:1108.4449] [InSPIRE].

[117] D.F. Litim, Optimized renormalization group flows, Phys. Rev. D 64 (2001) 105007 [hep-th/0103195] [INSPIRE].

[118] H. Gies and M.M. Scherer, Asymptotic safety of simple Yukawa systems, Eur. Phys. J. C 66 (2010) 387 [arXiv: 0901.2459] [INSPIRE]. 